\title{
Kinematics and properties of the central molecular zone as probed with [C II]
}

\author{
W. D. Langer ${ }^{1}$, T. Velusamy ${ }^{1}$, M. R. Morris ${ }^{2}$, P. F. Goldsmith ${ }^{1}$, and J. L. Pineda ${ }^{1}$ \\ 1 Jet Propulsion Laboratory, California Institute of Technology, 4800 Oak Grove Drive, Pasadena, CA 91109, USA \\ e-mail: William.Langer@jpl.nasa.gov \\ 2 Department of Physics and Astronomy, UCLA, 403 Portola Plaza, Los Angeles, CA 90095-1547, USA
}

Received 8 August 2016/ Accepted 12 December 2016

\begin{abstract}
Context. The Galactic central molecular zone (CMZ) is a region containing massive and dense molecular clouds, with dynamics driven by a variety of energy sources including a massive black hole. It is thus the nearest template for understanding physical processes in extragalactic nuclei. The CMZ's neutral interstellar gas has been mapped spectrally in many neutral atomic and molecular gas tracers, but the ionized and $\mathrm{CO}$-dark $\mathrm{H}_{2}$ regions are less well traced spectroscopically.

Aims. We identify features of the UV irradiated neutral gas, photon dominated regions (PDRs) and CO-dark $\mathrm{H}_{2}$, and highly ionized gas in the $\mathrm{CMZ}$ as traced by the fine structure line of $\mathrm{C}^{+}$at $158 \mu \mathrm{m},[\mathrm{C} \mathrm{II}]$, and characterize their properties.

Methods. We observed the [C II] $158 \mu \mathrm{m}$ fine structure line with high spectral resolution using Herschel HIFI with two perpendicular on-the-fly strip scans, along $l=-0.8$ to +0.8 and $b=-0.8$ to +0.8 , both centered on $(l, b)=\left(0^{\circ}, 0^{\circ}\right)$. We analyze the spatial-velocity distribution of the $[\mathrm{C} \mathrm{II}]$ data, compare them to those of $[\mathrm{CI}]$ and $\mathrm{CO}$, and to dust continuum maps, in order to determine the properties and distribution of the ionized and neutral gas and its dynamics within the CMZ.

Results. The longitude- and latitude-velocity maps of [C II] trace portions of the orbiting open gas streams of dense molecular clouds, the cloud G0.253+0.016, also known as the Brick, the arched filaments, the ionized gas within several pc of Sgr A and Sgr B2, and the warm dust bubble. We use the [C II] data to determine the physical and dynamical properties of these CMZ features.

Conclusions. The bright far-IR $158 \mu \mathrm{m} \mathrm{C}^{+}$line, [C II], when observed with high spatial and spectral resolution, traces a wide range of emission features in the CMZ. The [C II] emission arises primarily from dense PDRs and highly ionized gas, and is an important tracer of the kinematics and physical conditions of this gas.
\end{abstract}

Key words. galaxies: ISM - Galaxy: center - ISM: clouds - ISM: atoms

\section{Introduction}

The Galaxy's central molecular zone (CMZ) is a roughly $400 \mathrm{pc} \times 100 \mathrm{pc}$ region stretching from $l \sim-1.0$ to $\sim+1.5$ that is a significantly different environment than the Galactic disk. Among many prominent features in the $\mathrm{CMZ}$ are a massive, $\sim 4 \times 10^{6} M_{\odot}$, black hole, the Galactic center bubble containing the arches and quintuplet clusters (Morris \& Serabyn 1996; Molinari et al. 2014), the radio arc and arched filaments (Yusef-Zadeh et al. 1984; Serabyn \& Guesten 1987; Yusef-Zadeh \& Morris 1987), and gas streams of dense molecular clouds orbiting the Galactic center at a radius of 100 to 120 pc (Tsuboi et al. 1999; Molinari et al. 2011; Jones et al. 2012; Kruijssen et al. 2015; Henshaw et al. 2016a,b). The CMZ molecular clouds contain about $10 \%$ of the Galaxy's molecular gas, have high average densities $\left(>10^{4} \mathrm{~cm}^{-3}\right)$, reside in a high thermal pressure environment, are relatively densely packed (Morris \& Serabyn 1996; Ferrière et al. 2007), and have regions with an intense flux of stellar far-UV radiation as well as X-rays (see review by Ponti et al. 2013). There is ample evidence of large-scale energetic gas motions in the CMZ, as indicated by the line widths of $\mathrm{CO}$ in giant molecular clouds (GMCs) of 10 to $20 \mathrm{~km} \mathrm{~s}^{-1}$, compared to a few $\mathrm{km} \mathrm{s}^{-1}$ in the Galactic disk (cf. Oka et al. 1998; Dame et al. 2001), and by the flows of material within and across this region (cf. Morris \& Serabyn 1996; Morris 1997; Enokiya et al. 2014). The CMZ produces $\sim 10 \%$ of the Galaxy's infrared luminosity, yet has a relatively low star formation rate, considering the mass of dense molecular gas there (Taylor et al. 1993; Longmore et al. 2013).

The CMZ's interstellar medium (ISM) has been fully or partially mapped spectrally in over twenty gas tracers, including several isotopologues (Morris 1997; Jones et al. 2012). The vast majority trace the neutral gas; these include the rotational transitions of CO isotopologues (e.g. Oka et al. 1998, 2011, 2012; Enokiya et al. 2014), CS (Tsuboi et al. 1999; Jones et al. 2012), HCN (Jackson et al. 1996; Jones et al. 2012), $\mathrm{N}_{2} \mathrm{H}^{+}$ (Jones et al. 2012), $\mathrm{H}_{2} \mathrm{CO}$ (Ginsburg et al. 2016), $\mathrm{CH}_{3} \mathrm{CCH}$ (Jones et al. 2012), as well as $\mathrm{NH}_{3}$ (Mills \& Morris 2013) and OH (Yusef-Zadeh et al. 1999), and the lower fine structure emission line of neutral carbon, [CI] (Martin et al. 2004). However, these species do not trace major components of the inner Galaxy, where the gas is highly ionized or where the UV irradiated neutral gas is weakly ionized. These regions include the photon dominated regions (PDRs), the ionized boundary layers (IBL) surrounding UV-irradiated molecular clouds, $\mathrm{H}$ II regions, $\mathrm{CO}-$ dark $\mathrm{H}_{2}$ clouds, and the warm and diffuse ionized gas. Less is known about these components due to the difficulty of observing key tracers of the ionized gas, the fine structure lines of $\mathrm{C}^{+}$ 
and $\mathrm{N}^{+}$, from the ground. In this paper we present new results on the properties of the ionized and neutral gas in the CMZ based on strip scans using the spectrally resolved fine structure line of ionized carbon.

Prior to the launch of the Herschel Space Observatory ${ }^{1}$ (Pilbratt et al. 2010) the CMZ had been mapped in the fine structure line of $\mathrm{C}^{+}$at $158 \mu \mathrm{m},[\mathrm{C} \mathrm{II}]$, with COBE FIRAS (Bennett et al. 1994) and the Balloon-borne Infrared Carbon Explorer (BICE; Nakagawa et al. 1998), but with its emission spectrally unresolved. However, with the Heterodyne Instrument in the Far-Infrared (HIFI; de Graauw et al. 2010) it became possible to map spectrally resolved [C II] in the CMZ. The Herschel HEXGAL programme (Güsten 2007) made a well sampled HIFI [C II] on-the-fly (OTF) map of a small region around Sgr A (García 2015; García et al. 2016) consisting of two slightly offset rectangles totaling $\sim 0.1$ square degrees in area. As part of the Herschel GOT C+ programme (Langer et al. 2010), [C II] was observed with HIFI along two spectrally resolved strip scans traversing 1.6 in longitude and latitude through $(l, b)=\left(0^{\circ}, 0^{\circ}\right)$. The purpose of this paper is to gain insight on the nature of the CMZ as traced by these two [C II] strip scans, which extend about half the length and the full width of the CMZ. We limit our discussion to describing the regions and features detected in [C II] emission, their kinematics, and estimates of the properties of the $\mathrm{C}^{+}$gas, as detailed information about the structure of these features is not possible without spectrally resolved $(l-b)$ maps well sampled in both longitude and latitude.

While Herschel HIFI provides the necessary spectrally resolved instrument to study [C II], the small beam size at $1.9 \mathrm{THz}$, $12^{\prime \prime}$, and single pixel made it unsuited to generate large-scale fully sampled maps, such as of the Galactic disk, CMZ, or external galaxies. However, Herschel HIFI was able to provide well sampled detailed maps of small regions or a coarse picture of [C II] emission across the Galaxy by conducting a large-scale sparse survey of spectrally resolved [C II].

The Herschel open time key programme, Galactic Observations of Terahertz C+ (GOT C+; Langer et al. 2010; Pineda et al. 2013) had two main subprogrammes: 1) a Galactic disk sparse pointed survey of [C II]; and, 2) a central molecular zone OTF strip scan survey of [C II]. In subprogramme (1) the GOT C+ survey characterized the entire Galactic disk by making a sparse sample covering $360^{\circ}$ in the plane. (The GOT C+ data sets are available as a Herschel User Provided Data Product under KPOT_wlanger_1.) The disk survey contains 500 lines of sight of spectrally resolved [C II] emission throughout the Galactic disk $\left(l=0^{\circ}\right.$ to $360^{\circ}$ and $\left.|b| \leq 1^{\circ}\right)$. The sampling in Galactic longitude was non-uniform in order to weight as best as possible a uniform angular volume across the disk, with an emphasis on the important inner longitude range $|l| \leq 90^{\circ}$. By analyzing a large sample of spectrally resolved components dispersed throughout the Galaxy, rather than large-scale maps of a few clouds, this survey allows a statistical approach to characterizing the ISM in [C II] and the results have been reported in a series of papers Langer et al. (2010), Pineda et al. (2010), Velusamy et al. (2010, 2012), Pineda et al. (2013), Langer et al. (2014), Pineda et al. (2014), Velusamy \& Langer (2014), Velusamy et al. (2015). Here we present the results of the GOT C+ [C II] strip scans of the CMZ, which pass through, or close to, several features, including Sgr A, G0.253+0.016 (the Brick), Sgr B2, the Galactic

\footnotetext{
1 Herschel is an ESA space observatory with science instruments provided by European-led Principal Investigator consortia and with important participation from NASA.
}

center warm dust bubble, the Radio Arc, the arched filaments, and the open streams of gas orbiting the Galactic center.

This paper is organized as follows. In Sect. 2 we describe the data reduction, while Sect. 3 presents the distribution of the [C II] traced gas compared to features in the CMZ. Section 4 discusses the properties of several features traced by [C II] in the $\mathrm{CMZ}$, and Sect. 5 summarizes the results.

\section{2. [C II] Observations and data reduction}

We observed the ionized carbon $\left(\mathrm{C}^{+}\right){ }^{2} \mathrm{P}_{3 / 2}{ }^{2} \mathrm{P}_{1 / 2}$ fine structure line, [C II], at $1900.5369 \mathrm{GHz}(\lambda \sim 157.74 \mu \mathrm{m})$, using HIFI in an OTF mode to produce position-velocity maps of the Galactic center region. We observed two strip scans through $(l, b)=$ $\left(0^{\circ}, 0^{\circ}\right)$, one along longitudes from $l=359.20$ to 0.80 at latitude $b=0^{\circ}$ and the other along $b=-0.80$ to +0.80 at longitude $l=0^{\circ}$; details of the observations and data reduction are given below.

\subsection{Observations}

All [C II] spectral line mapping observations were made with the high spectral resolution HIFI instrument (de Graauw et al. 2010) onboard Herschel (Pilbratt et al. 2010) in February and March 2011. These [C II] spectral line map scans used the HIFI band $7 \mathrm{~b}$ and the wide band spectrometer (WBS). The longitude scan consisted of four OTF scans taken along Galactic longitude centered on $(l, b)=(0.6,0 \circ 0),(0 \circ 2,0.0),(359: 8,0.0)$, and $(359 \circ 4,0.0)$. Similarly, the latitude scan consisted of four OTF scans taken in Galactic latitude centered on $(l, b)=(0.0,-0.6)$, $(0.0,-0.2),(0 \circ 0,+0 \circ 2)$, and $(0 \circ 0,+0 \circ 6)$. Each OTF scans was 24 arcmin long, thus the four together cover a range of $1.6^{\circ}$. The observing duration of each OTF scan was $\sim 2500 \mathrm{~s}$ and the 24 arcmin-long scan data were read out every $40^{\prime \prime}$ (i.e. averaged over a $40^{\prime \prime}$ window).

All HIFI OTF scans were made in the LOAD-CHOP mode using a reference off-source position about $2^{\circ}$ away in latitude (this HIFI observing mode requires the reference position be within $2^{\circ}$ of the target). For all four $l$-scans and the two $b$-scans centered at $b \geq 0.0$ we used the $\operatorname{OFF}(l, b)=(0.0,1.9)$ which is very clean of [C II] emission at the sensitivity of the survey. For the $b$-scans at $b<0.0$ we had to use a reference position closer to the plane at $(l, b)=(0.0,0.5)$ which contains [C II] emission. However, this reference position has been well observed in our HIFI Pointed observation programme using the reference sky position at $(0.0,+1.9)$ so that the $[\mathrm{C}$ II] emission spectrum at this position is well characterized and can be used as a sky reference to correct for OFF source emission. Thus the mapping scheme used for the $\mathrm{CMZ}$ provides [C II] spectral line map data all of which are effectively referenced back to a common position at $(l, b)=(0.0,1: 9)$. The GOT C+ OTF scan reference spectra extracted in HIPE-13, show little emission at a $3 \sigma$ level of $\sim 0.1 \mathrm{~K}$.

Near the Galactic center we expect [C II] emission over a wide range of velocities $\left(>200 \mathrm{~km} \mathrm{~s}^{-1}\right)$. However, the useful instantaneous velocity range observable with HIFI band $7 \mathrm{~b}$ is $\sim 160 \mathrm{~km} \mathrm{~s}^{-1}$. To accommodate this velocity range and extract the spectral line data, each OTF scan was observed at two local oscillator (LO) frequencies with frequency offset of $0.9 \mathrm{GHz}$ (corresponding spectra centered at $V_{\mathrm{LSR}}=-80 \mathrm{~km} \mathrm{~s}^{-1}$ and $+70 \mathrm{~km} \mathrm{~s}^{-1}$ ).

\subsection{Data reduction}

We processed the OTF strip scan data and produced the $l$ - and $b$-scan position-velocity maps, $(l-V)$ and $(b-V)$, following the 
procedure discussed in Velusamy et al. (2015). However, the results presented here were processed in HIPE-13, rather than HIPE-12 used in Velusamy et al. (2015), taking advantage of the hebCorrection tool, which removes the HEB standing waves present in band $7 \mathrm{~b}$ data. hebCorrection is applied in HIPE-13 as part of the pipeline to extract spectra at the reference sky position. From the Level 2 data, the [C II] strip scans were made into "spectral line cubes" using the standard mapping scripts in HIPE. Any residual HEB and optical standing waves in the reprocessed Level 2 data were minimized by applying fitHifiFringe to the "gridded" spectral data. We took the additional precaution in fitHifiFringe of disabling DoAverage in order not to bias the spectral line window.

The H- and V-polarization data were processed separately and were combined only after applying fitHifiFringe to the gridded data. This approach minimizes the standing wave residues in the strip scans by taking into account the standing wave differences between $\mathrm{H}$ - and V-polarization. In view of the fact that the spectrum of a single LO observation may not provide adequate baselines at both ends of the spectral line profile in HIPE-13, we generated two sets of spectral cubes with and without baseline subtraction. We then used the processed spectral line data cubes in two polarizations ( $\mathrm{H}$ and $\mathrm{V}$ ) and two LO settings to make $(l-V)$ and $(b-V)$ maps, by combining them after fitting baseline and velocity offsets. For $|l|>0.4$ and $|b|>0.4$ combining the spectral cube data was straightforward as these spectra had clean baselines at both ends in one or both sets of the two LO settings.

However, for $|l|<0.4$ and $|b|<0.4$ the spectra contained a long and clean baseline only at one end, with emission present at the opposite end. In this case the spectra of the LO-pairs were combined, fixing the linear baseline at the cleaner end and matching the profiles at the overlapping velocities. In Fig. 1 we show an example of this procedure for $l=0.06$, where it can be seen that the matching produces a good baseline correction. For matching we use the region near $V_{\mathrm{LSR}} \sim 0 \mathrm{~km} \mathrm{~s}^{-1}$ where [C II] is absorbed by foreground emission. To check this approach, we compared the resulting spectra for $|l|>0.4$, where the emission is narrow enough to fit within the band of a single LO, and the baselines can be set at both ends with no matching of profiles. The resulting baselines and spectra fit both ways are in good agreement, thus lending confidence that the matching approach produces good baselines for $|l|<0.4$ and $|b|<0.4$. We find that this matching procedure does introduce a baseline uncertainty of $\sim 0.5 \mathrm{~K}$ and produces more uncertainty in the final spectra for the $l$ scan than for $b$ above and below the plane because these $b$ scans generally have narrower lines and, therefore longer and cleaner baselines.

At $1.9 \mathrm{THz}$ the angular resolution of the Herschel telescope is $12^{\prime \prime}$, but the [C II] OTF observations were averaged over a $40^{\prime \prime}$ window along the scan direction (i.e. read out at $40^{\prime \prime}$ intervals). Such fast scanning broadens the effective beam size along the scan direction (Mangum et al. 2007). Therefore all [C II] maps have been restored with effective beam sizes corresponding to twice the sample averaging interval along the scan direction $\left(\sim 80^{\prime \prime}\right)$. We used the HIFI Wide Band Spectrometer (WBS) with a spectral resolution of $1.1 \mathrm{MHz}\left(0.17 \mathrm{~km} \mathrm{~s}^{-1}\right)$ for all the scan maps. The final $l-V$ and $b-V$ maps presented here were smoothed to a velocity resolution of $2 \mathrm{~km} \mathrm{~s}^{-1}$. The rms in the main beam temperature, $T_{\mathrm{mb}}$, is in the range 0.21 to $0.31 \mathrm{~K}$ for the $l-V$ map and 0.18 to $0.22 \mathrm{~K}$ for the $b$ - $V$ map, the smaller rms for the $b$ $V$ map is due to the cleaner baseline that results from having (generally) narrower line profiles along $b$ than along $l$.

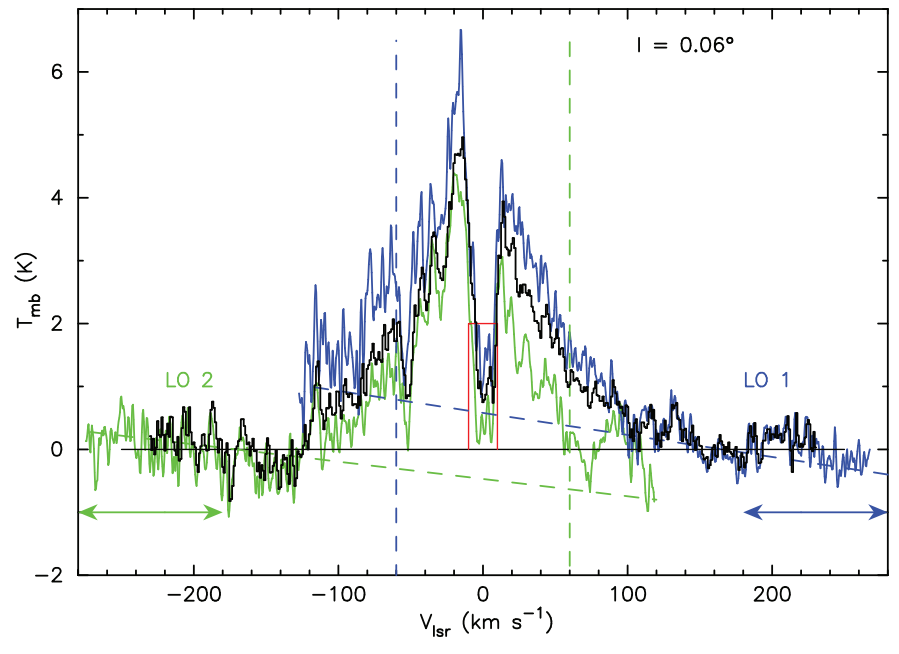

Fig. 1. Example of combining the two LO spectra in OTF scan maps. The spectra at $l=0.06$ in the individual longitude scan maps consist of two local oscillator settings, LO 1 (blue) and LO 2 (green). The final averaged spectrum is shown in black. The double arrows mark the velocity range of a constant baseline offset used in the corresponding maps. The red box denotes the velocity range $\left( \pm 10 \mathrm{~km} \mathrm{~s}^{-1}\right)$ for matching the two spectra and the baseline offsets here were estimated using their averaged intensity. The quasi-horizontal dashed lines represent a linear fit to the baselines used for the spectra for final averaging. The vertical dashed lines mark velocity limits above (green) and below (blue) where the data are unusable (near the edges of the band pass). The spectra for velocities within $\pm 60 \mathrm{~km} \mathrm{~s}^{-1}$ were averaged with baseline corrections for $V<-60$ and $V>+60 \mathrm{~km} \mathrm{~s}^{-1}$ for LO 2 and LO 1, respectively. Note that the absorption feature around $0 \mathrm{~km} \mathrm{~s}^{-1}$ is due to local gas.

\section{Results}

In this section we present the [C II] OTF strip scans and auxiliary spectral line maps of $[\mathrm{CI}]$ and $\mathrm{CO}$, and describe their morphological relationships to $\mathrm{CMZ}$ features.

\subsection{OTF maps}

In Fig. 2 we show our HIFI GOT C+ position-velocity [C II] maps along with corresponding Herschel $70 \mu \mathrm{m}$ dust and H I emission maps from Molinari et al. (2011). In Fig. 2a we plot the longitude-velocity, $l-V,[\mathrm{C} \mathrm{II}]$ emission and in Fig. $2 \mathrm{~d}$ the latitude-velocity, $b$ - $V$, emission. The velocity dispersion along both the longitude and latitude strips is largest near $(l, b)=$ $\left(0^{\circ}, 0^{\circ}\right)$ and the highest detected velocities, as outlined by the white rectangles, reach about $\pm 150 \mathrm{~km} \mathrm{~s}^{-1}$. The locations in position-velocity space corresponding to a few features in the CMZ, including Sgr B2, the Brick, the arched filaments, and the open orbit streams of clouds are marked on the $l$ - $V$ plot. The lowintensity vertical striations in Fig. $2 \mathrm{a}$ in the $l$-scan are artifacts of the baseline uncertainties. These features are less prominent in the $b$-scan, as seen in Fig. $2 \mathrm{~b}$, because the baseline uncertainties are smaller (see Sect. 2.2).

We have also highlighted the ellipse (red solid line) in $l$ - $V$ at $b=0^{\circ}$ corresponding to the kinematics of a twisted elliptical ring at $b=0^{\circ}$ which Molinari et al. (2011) used to explain the distribution of CS velocities in Fig. 2b. The dashed (red) ellipse is our fit to the [C II] emission and will be discussed in Sect. 4.1. Panel b shows an atomic hydrogen column density spatial map of the CMZ with velocities from CS superimposed along portions of the elliptical ring from Molinari et al. (2011). Panel c shows the extent of the two OTF strip scans (dashed white lines) 


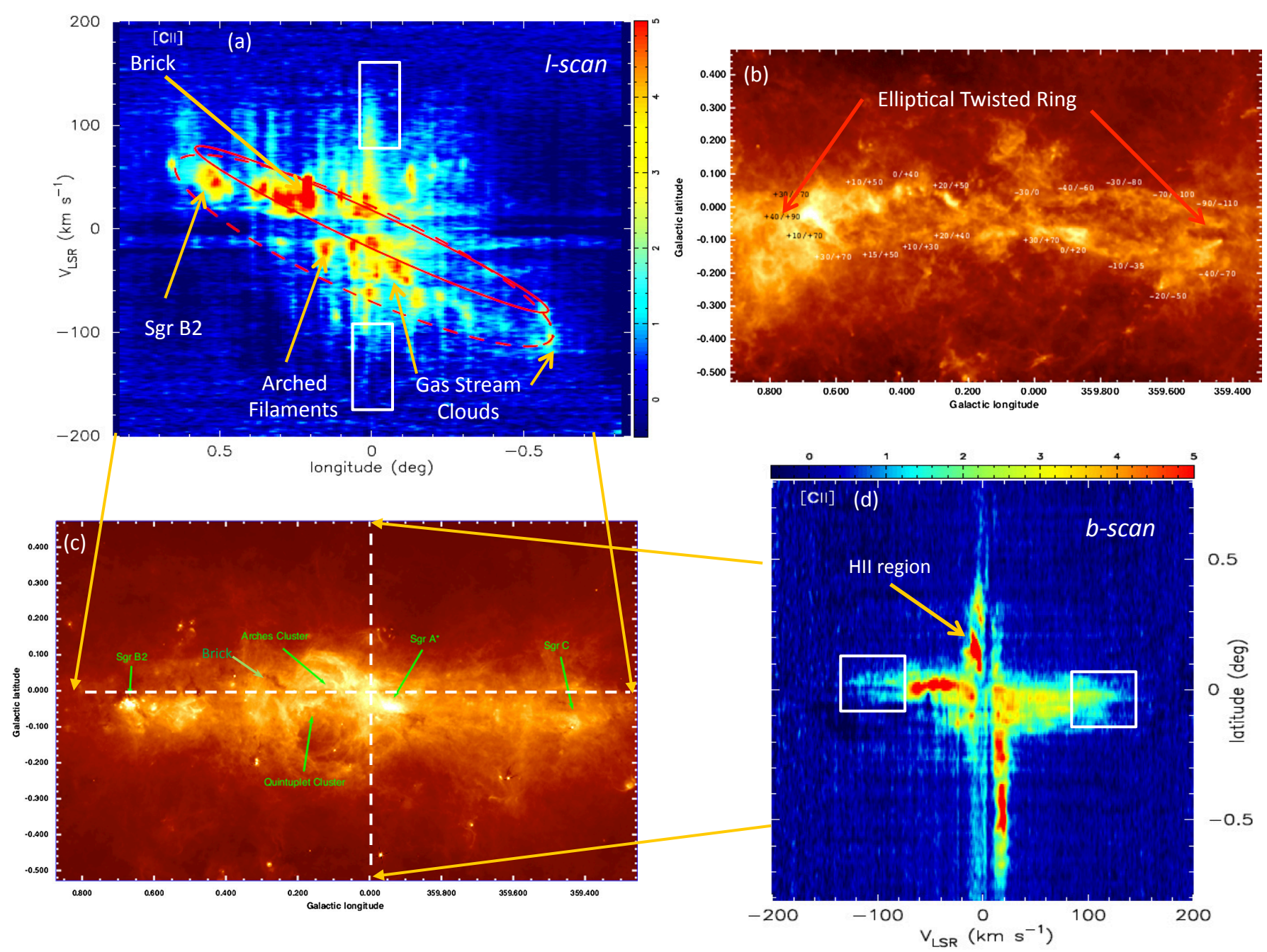

Fig. 2. HIFI position-velocity [C II] maps of the CMZ tracing several notable features, including the Brick, open orbit streams of molecular gas, Sgr B2, and the arched filament. a) The [C II] longitude-velocity map, $l-V$, covering 1.6 centered at $\left(0^{\circ}, 0^{\circ}\right)$. The color bar indicates the [C II] main beam temperature, $T_{\mathrm{mb}}(\mathrm{K})$. The red-solid ellipse is the solution at $z=0: 0$ for the elliptical ring model from Molinari et al. (2011) in longitudevelocity space and the red-dashed ellipse is our fit to the [C II] observations assuming an ellipse. The vertical striations are artifacts of the baseline uncertainties (see Sect. 2). b) Atomic hydrogen column density map from Molinari et al. (2011) highlighting their elliptical twisted ring model. The line-of-sight velocity along the ring was derived by Molinari et al. (2011) from CS observations and is marked along the ring (see the original figure). c) A Herschel PACS $70 \mu \mathrm{m}$ image of the CMZ from Fig. 1 of Molinari et al. (2011). The [C II] OTF strip scans in $l$ and $b$ are indicated by white dashed lines. The labels in green are from Molinari et al. (2011), except for the Brick (G0.253+0.016). d) The [C II] latitude-velocity map, $b-V$, covering 1.6 centered at $\left(0^{\circ}, 0^{\circ}\right)$. The color bar indicates $T_{\mathrm{mb}}([\mathrm{C} \mathrm{II}])$ in degrees $\mathrm{K}$. In both $l-V($ panel a)) and $b-V$ (panel d)) we highlight the position-velocity extent of the high velocity gas near the center (white boxes). The arrow indicates a very bright [C II] peak that is likely a foreground H II source (see Sect. 4.8).

superimposed on a Herschel $70 \mu \mathrm{m}$ map from Molinari et al. (2011). Figure $2 \mathrm{~d}$ plots the $b-V[\mathrm{CII}]$ emission at $l=0^{\circ}$. The velocity gradient is small in this map compared to that of the $l-V$ map.

In Fig. 3 we show the central portion of the dust temperature map of the CMZ from Fig. 3 in Molinari et al. (2011), which was derived from the Herschel Hi-GAL 70-350 $\mu$ m survey (see also Fig. 5 in Molinari et al. 2014). The Galactic center bubble is surrounded by warm dust with a doughnut-like temperature distribution seen in projection, located to the left and below the Galactic center. The central portion of the doughnut is an ionized gas bubble centered at about $(0.11,-0 \circ 11)$. The dust temperature map shown in Fig. 3 covers about 0.76 in longitude and 0.96 in latitude. The white dashed lines are the [C II] OTF strip scans. The [C II] longitudinal strip scan goes through the warm dust doughnut, while the latitudinal strip scan appears to be at the edge of the warm dust doughnut. Also shown is a radio continuum map of the innermost portion of the $\mathrm{CMZ}$ (Yusef-Zadeh \& Morris 1987; Simpson et al. 2007) revealing the Radio Arc, the arched filaments, and the clusters of massive stars associated with the Arches and Quintuplet Clusters. The red dashed lines designate the [C II] OTF strip scans through this region and it can be seen that the [C II] longitude strip scan goes through the arched filaments and passes near the Arches Cluster.

The arched filaments have mostly negative velocities in the range -70 to $+15 \mathrm{~km} \mathrm{~s}^{-1}$ (Lang et al. 2001; Simpson et al. 2007). As can be seen in Fig. 2a, [C II] is detected along the line-of-sight near the base of the arched filaments, $l \sim 0.06$ (see Figs. 1 in Lang et al. 2001, 2002) with a velocity range within those of the filaments. It is also detected along $l \sim 0.16$, that might also be associated with the arched filaments, but is more likely associated with the molecular clouds with $V_{\mathrm{LSR}} \sim-20$ to 


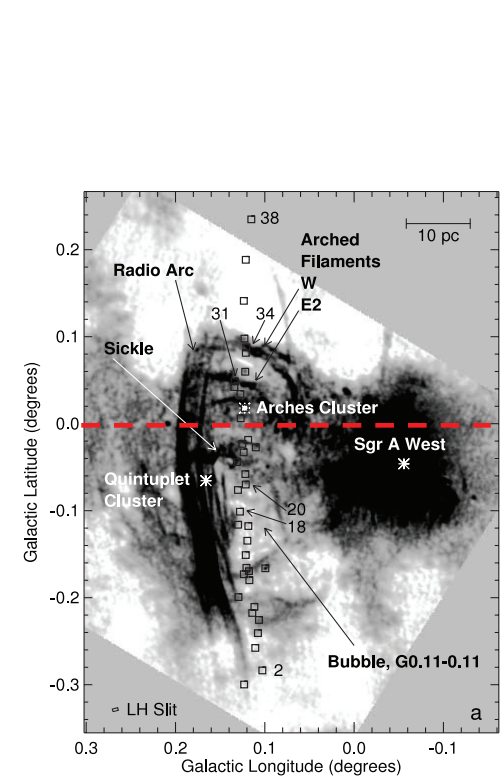

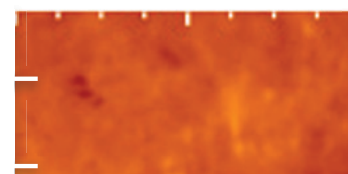
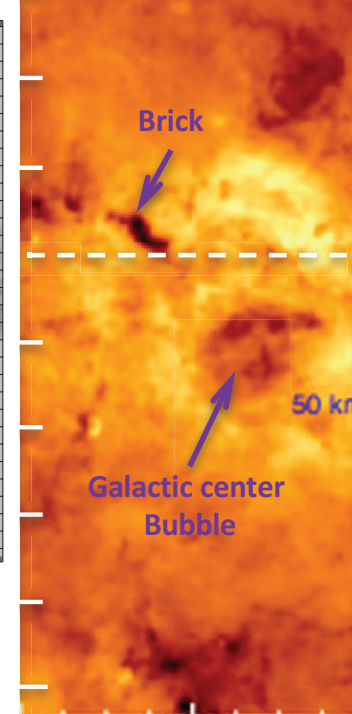

0.2

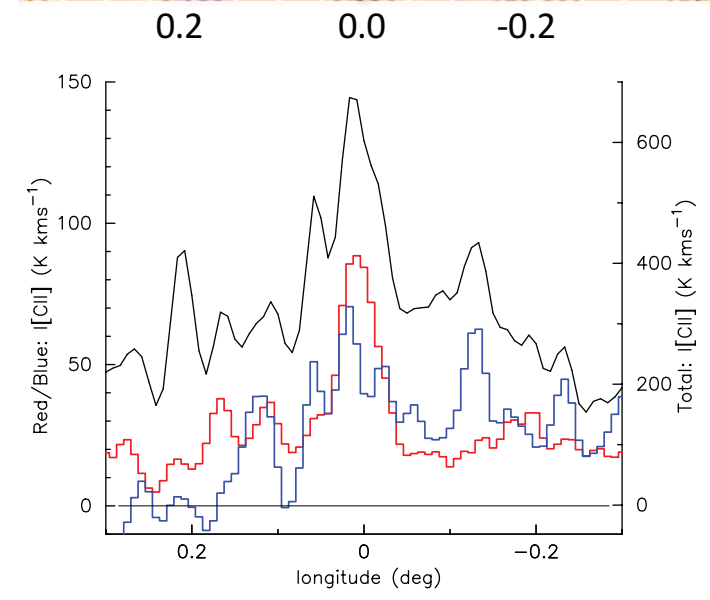

0.2

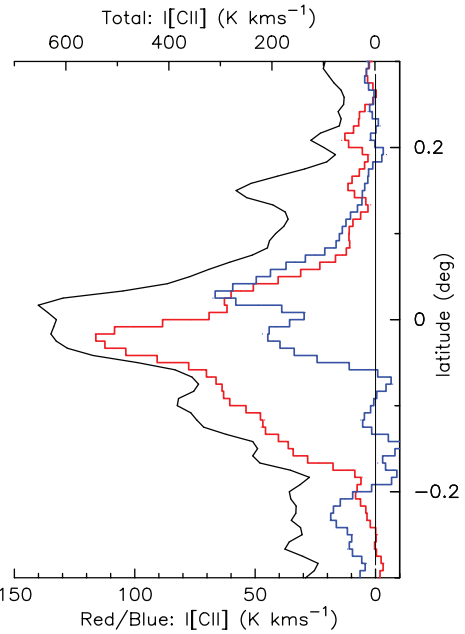

\section{0}

(1)

Fig. 3. The center image is the dust temperature distribution of the inner CMZ derived from Herschel HI-GAL 70-350 $\mu \mathrm{m}$ images (Molinari et al. 2011). It has a doughnut shaped distribution as seen in projection marking a warm dust bubble (Molinari et al. 2011, 2014). On the left is the radio continuum map generated by Simpson et al. (2007) based on the data of Yusef-Zadeh \& Morris (1987) of the central portion of the bubble (the hole in the doughnut) containing the Radio Arc and arched filaments. The [C II] integrated intensity, $I([\mathrm{C}$ II $])\left(\mathrm{K} \mathrm{km} \mathrm{s}^{-1}\right)$, strip scans as a function of longitude and latitude are plotted to the bottom and the right, respectively, and are aligned with the temperature map of the inner CMZ. These plots show the velocity-integrated [C II] intensity profiles as functions of longitude or latitude: the black lines represent the total intensity in the velocity range $V_{\mathrm{LSR}}=-200$ to $+140 \mathrm{~km} \mathrm{~s}^{-1}$, the red and blue lines represent $I([\mathrm{C} \mathrm{II}])$ integrated emission in the high-velocity red and blue wings from $V_{\mathrm{LSR}}=+80$ to $+130 \mathrm{~km} \mathrm{~s}^{-1}$ and -80 to $-130 \mathrm{~km} \mathrm{~s}^{-1}$, respectively.

$-40 \mathrm{~km} \mathrm{~s}^{-1}$. There is also [C II] emission at $l \sim 0.20$ and with velocities $\sim+20$ to $+30 \mathrm{~km} \mathrm{~s}^{-1}$ that is probably associated with the molecular cloud M0.20-0.033 (Serabyn \& Morris 1994).

In Fig. 3 we plot the total integrated intensity $I([\mathrm{C}$ II $])=$ $\int T_{\mathrm{mb}} \mathrm{d} v\left(\mathrm{~K} \mathrm{~km} \mathrm{~s}^{-1}\right)$ over the velocity range -200 to $+140 \mathrm{~km} \mathrm{~s}^{-1}$ (black lines) as a function of longitude and latitude, bottom and right, respectively. We also plot the integrated [C II] intensity, $I([\mathrm{C}$ II $])$, as a function of longitude and latitude for two different velocity ranges. The $V_{\mathrm{LSR}}$ velocity range is 80 to $130 \mathrm{~km} \mathrm{~s}^{-1}$ (red) away from and -80 to $-130 \mathrm{~km} \mathrm{~s}^{-1}$ (blue) towards the sun. The $l$ - and $b$-scan intensity profiles in Fig. 3 indicate significantly large [C II] emission present at the lower velocities $\left(\left|V_{\mathrm{LSR}}\right|<80 \mathrm{~km} \mathrm{~s}^{-1}\right)$ in comparison to that in the high velocity wings $\left(\left|V_{\mathrm{LSR}}\right|>80 \mathrm{~km} \mathrm{~s}^{-1}\right)$. The strip scan in latitude shows a shift in $b$ in the peak [C II] emission between the blue and redshifted components. A likely explanation for the latitude offset between these two velocity regimes is the tilt in the CMZ (Morris \& Serabyn 1996, and references therein).

In Fig. 4 we compare the gas in the $\mathrm{CMZ}$ traced by $\mathrm{C}^{+}$with their neutral cloud components, $\mathrm{C}$ and $\mathrm{CO}$, using the AST/RO maps of $[\mathrm{CI}]{ }^{2} \mathrm{P}_{1} \rightarrow{ }^{2} \mathrm{P}_{0}$ and $\mathrm{CO}(4-3)$ (Martin et al. 2004). The AST/RO spatial maps cover a longitude range -1.3 to +2.0 , larger than the GOT C+ OTF strip scan, but were limited in latitude to -0.3 to +0.2 , smaller than the GOT $\mathrm{C}+$ strip scan. The AST/RO maps have an angular resolution $\sim 120^{\prime \prime}$, which is about $50 \%$ larger along the scan direction than the effective resolution in the GOT C+ strip scans. The AST/RO spectra were resampled to match the velocity resolution of $2 \mathrm{~km} \mathrm{~s}^{-1}$ in the GOT C+ [C II] OTF scans. $\mathrm{CO}(4-3)$ and $[\mathrm{CI}]$ in the AST/RO maps are strong at $b<0^{\circ}$ (see Fig. 3 in Martin et al. 2004) and relatively weak at 

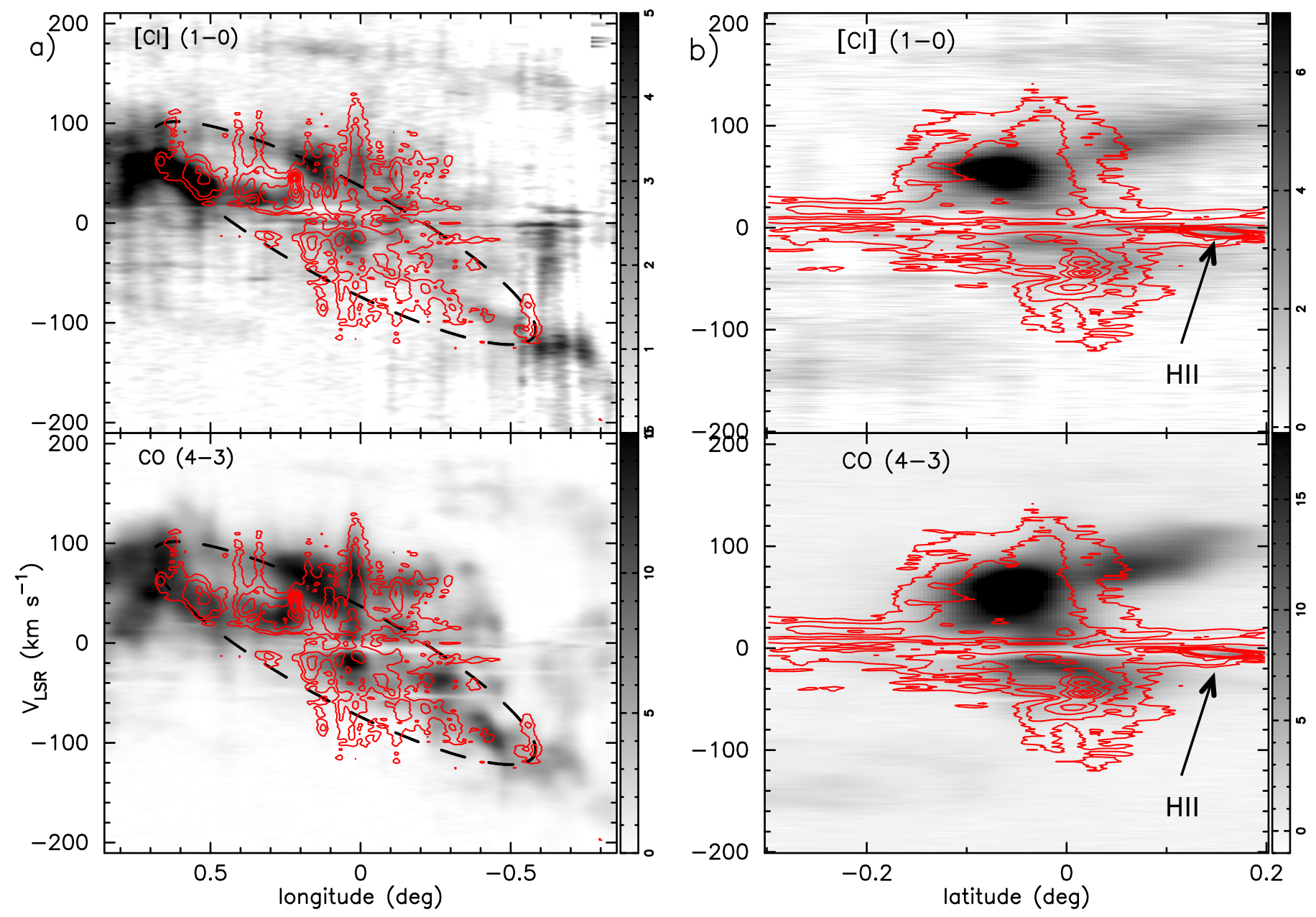

Fig. 4. Comparison of the $[\mathrm{C} \mathrm{II}]$ position-velocity strip scans with the AST/RO observations of $[\mathrm{CI}]$ and $\mathrm{CO}(4-3)$ (Martin et al. 2004). a) The [C II] longitude-velocity strip scan and b) the latitude-velocity strip scan. The [CI] and CO maps are shown in gray scale, where the grey scale bars on the right hand side indicate the main beam temperatures, $T_{\mathrm{mb}}(\mathrm{K})$; the corresponding $[\mathrm{C} \mathrm{II}]$ emission maps are overlaid as contours. The [C II] contour levels (red) are $T_{\mathrm{mb}}(\mathrm{K})=1,2,4,6,8$, and 10. In the $l-V$ maps the dashed line roughly outlines the [C II] emission at $b=0.0$ for the elliptical ring model of Molinari et al. (2011; see also Fig. 2). This ellipse is used only to delineate the $l-V$ space where the open orbit gas streams can be found, and is not intended as a model of the gas dynamics. The arrows in panel b) indicate a local H II source (see Sect. 4.8).

$b>0^{\circ}$. The left panels show the $l-V$ maps and the right panels the $b$ - $V$ maps. The features in these strip scans will be discussed in more detail in Sect. 4.

The $l-V$ maps (left panels) of all three tracers show the same general trend in velocity with longitude, but the velocity dispersion near $l=0^{\circ}$ is larger in [C II] than in [C I] or $\mathrm{CO}(4-3)$. A strong peak in the [C II] longitude strip scan occurs at $l \sim 0.25$, corresponding to the location of the Brick. In addition there are some notable anti-correlations within the $l-V$ maps, some features have strong [C II] but weak or no [C I] and/or CO, for example at $l \sim 0.25$, and vice-versa. In the latitude-velocity maps (right panels) $[\mathrm{CI}]$ and $\mathrm{CO}(4-3)$ are generally correlated and, again, the $[\mathrm{C} \mathrm{II}]$ emission is weak where $[\mathrm{CI}]$ and $\mathrm{CO}(4-3)$ are strong, and vice-versa. We also note a strong [C II] feature at $b \sim 0.15$ which is a local H II region (see Sect. 4.8). Finally, the two local peaks in $[\mathrm{C}$ II] centered at $l \sim 0.06$ and 0.18 with $V_{\mathrm{LSR}} \sim-20$ to $-40 \mathrm{~km} \mathrm{~s}^{-1}$ appear associated with the arched filaments and Radio Arc filaments, respectively, but have little [C I] or $\mathrm{CO}(4-3)$ associated with them.

The absence of a strong correlation between [C II] and [C I] and between [C II] and $\mathrm{CO}(4-3)$ seen in many positions in Fig. 4 indicates that a significant fraction of the $[\mathrm{C} \mathrm{II}]$ emission in the
CMZ is tracing a highly ionized gas component. Furthermore, there is a trend for the highly ionized gas to appear closer to the Galactic center than further out from it. To explore this trend further and to look for any differences in the source of [C II] emission along the $\mathrm{CMZ}$, we evaluated the global relative intensity distribution along the Galactic longitude strip scan of [C II] with respect to $\mathrm{CO}(4-3)$ and $[\mathrm{C} \mathrm{I}]$ by computing their average integrated intensities, $I=\left\langle\left(\int T \mathrm{~d} v\right)\right\rangle$, in bins of 0.5 width. The average intensity in each bin was calculated using a velocity range of $\pm 130 \mathrm{~km} \mathrm{~s}^{-1}$ but excluding the range -10 to $+10 \mathrm{~km} \mathrm{~s}^{-1}$ which is contaminated with emission and absorption from local gas. The results are summarized in Table 1. Note that the $[\mathrm{C} \mathrm{II}] / \mathrm{CO}(4-3)$ ratio at the center bin, within \pm 0.25 of $(0.0,0 \circ 0)$, is about 2.3 times that on either side. Furthermore, the $[\mathrm{C} \mathrm{II}] /[\mathrm{CI}]$ ratio at the center bin is also about 3 times that on either side. Thus the results in Table 1 clearly show that there is a distinct excess in [C II] over $\mathrm{CO}(4-3)$ and [C I] within \pm 0.25 of the Galactic center. Unlike $\mathrm{CO}$ or [CI], the [C II] emission can arise in both the neutral medium, excited by collisions with $\mathrm{H}_{2}$ molecules, and in the ionized gas, excited by collisions with electrons. Therefore, by comparison with the regions outside the central bins, we can conclude that the $[\mathrm{CII}]$ emission within 
W. D. Langer et al.: The central molecular zone as probed with [C II]

Table 1. Longitudinal [C II] versus $\mathrm{CO}(4-3)$ and $[\mathrm{CI}]$ in the $\mathrm{CMZ}$.

\begin{tabular}{cccccc}
\hline \hline $\begin{array}{c}\text { Longitude } \\
\text { range }\end{array}$ & $\begin{array}{c}\langle I([\mathrm{C} \mathrm{II}])\rangle^{a} \\
\left(\mathrm{~K} \mathrm{~km} \mathrm{~s}^{-1}\right)\end{array}$ & $\begin{array}{c}\left.\left\langle I\left(\mathrm{CO}(4-3)^{b}\right)\right)\right\rangle^{a} \\
\left(\mathrm{~K} \mathrm{~km} \mathrm{~s}^{-1}\right)\end{array}$ & $\begin{array}{c}\left\langle I([\mathrm{C} \mathrm{I}])^{b}\right\rangle^{a} \\
\left(\mathrm{~K} \mathrm{~km} \mathrm{~s}^{-1}\right)\end{array}$ & $\langle I([\mathrm{C} \mathrm{II}])\rangle /\langle I(\mathrm{CO}(4-3))\rangle$ & $\langle I([\mathrm{C} \mathrm{II}])\rangle /\langle I([\mathrm{C} \mathrm{I}])\rangle$ \\
\hline 0.75 to 0.25 & 123 & 1012 & 345 & 0.12 & 0.36 \\
0.25 to -0.25 & 313 & 1013 & 269 & 0.31 & 1.16 \\
-0.25 to -0.75 & 67 & 570 & 172 & 0.12 & 0.38 \\
\hline
\end{tabular}

Notes. ${ }^{(a)}$ Average intensity calculated over the velocity range $\pm 130 \mathrm{~km} \mathrm{~s}^{-1}$ but excluding the emission in the range $\pm 10 \mathrm{~km} \mathrm{~s}^{-1}$ where it is most likely to be contaminated with emission from local gas. ${ }^{(b)}$ AST/RO observations from Martin et al. (2004).

\pm 0.25 of the Galactic center has a significant contribution due to electron excitation of highly ionized gas. This result is further corroborated by the detection of $[\mathrm{N}$ II] in the Galactic center (Goldsmith et al. 2015; García et al. 2016; Langer et al. 2016).

\section{2. [C II] spectra}

In Fig. 5 we show six [C II] spectra extracted from the OTF observations. The spectra at $(0.51,0.0)$ and $(-0.58,0.0)$ are at the hypothetical edges of the open orbit gas streams, $(-0.085,0.0)$ is $\sim 8$ pc outside Sgr A, and $(0.0,-0.20)$ and $(0.0,-0.49)$ show that $[\mathrm{C} \mathrm{II}]$ emission is significantly narrower below the plane. Within each spectrum there are narrow features with linewidths on the order of 10 to $15 \mathrm{~km} \mathrm{~s}^{-1}$, along with broad but weaker [C II]. In addition, there is evidence of absorption by local gas near $0 \mathrm{~km} \mathrm{~s}^{-1}$ in the innermost spectrum at $(-0.085,0.0)$.

At $(l, b)=\left(0^{\circ}, 0^{\circ}\right)$, in addition to a pointed observation of [C II] from GOT $\mathrm{C}+$, we also have a spectrum of the [N II] $205 \mu \mathrm{m}$ fine structure line taken from a Herschel HIFI survey of ten GOT C+ lines of sight (Goldsmith et al. 2015; Langer et al. 2016). The angular resolution for the [N II] spectra is $16^{\prime \prime}$ compared to $12^{\prime \prime}$ for [C II]. In Fig. 6 the [N II] spectrum is overlaid on the $[\mathrm{CII}]$ spectrum $^{2}$. It can be seen that there is emission across most of the velocity range of the [C II], but only one strong component. The line of sight to the Galactic center intercepts a number of spiral arms. We have indicated three features likely associated with the $3-\mathrm{kpc}$ and $4.5-\mathrm{kpc}$ arms, and local gas. The strong peak in [N II] aligns with the [C II] peak towards the 3-kpc arm and likely most of the emission arises there. A Gaussian fit to this [N II] spectral feature yields $V_{\mathrm{LSR}} \sim-60 \mathrm{~km} \mathrm{~s}^{-1}$ and a FWHM line width $\sim 17 \mathrm{~km} \mathrm{~s}^{-1}$. The strongest [C II] feature has very similar line parameters, $V_{\mathrm{LSR}} \sim-64 \mathrm{~km} \mathrm{~s}^{-1}$ and $F W H M \sim 19 \mathrm{~km} \mathrm{~s}^{-1}$. The couple of $\mathrm{km} \mathrm{s}^{-1}$ shift in $V_{\mathrm{LSR}}$ between [C II] and [N II] is characteristic of the emission arising from different regions across the spiral arm profile (Velusamy et al. 2015). The remaining [N II] is broad low level emission arising from the CMZ and it is difficult to extract any distinct spectral lines with Gaussian-like shape. The $4.5 \mathrm{kpc}$ arm aligns with a peak in [C II]. The absorption by the local arm and local foreground gas at $V_{\mathrm{LSR}} \sim-10$ to $+10 \mathrm{~km} \mathrm{~s}^{-1}$, which is possibly in the Riegel-Crutcher cold cloud (Riegel \& Crutcher 1972), and which is very evident in [C II], but is not apparent in the [N II] spectrum. The $\mathrm{N}^{+}$abundance is a factor of a few less than $\mathrm{C}^{+}$ and probably has too low an opacity to absorb significantly the [N II] emission from the CMZ.

\footnotetext{
2 We reproduce the [C II] and [N II] spectra from Langer et al. (2016) here because the OTF strip scans show the spatial extent of the [C II] component associated with [N II] in the 3-kpc arm.
}

The [N II] line can be used to separate the contributions of [C II] from highly ionized gas and neutral gas (PDRs and $\mathrm{CO}$-dark $\mathrm{H}_{2}$ ). The [C II] intensity from the fully ionized gas, $I_{\text {ion }}([\mathrm{C} \mathrm{II}])$, is approximately proportional to the $[\mathrm{N} \mathrm{II}]$ intensity over a wide range of temperature and electron density, $I_{\text {ion }}([\mathrm{C} \mathrm{II}]) \sim 0.9\left(\mathrm{C}^{+} / \mathrm{N}^{+}\right) I([\mathrm{~N} \mathrm{II}])$, where $\left(\mathrm{C}^{+} / \mathrm{N}^{+}\right)$is the carbon to nitrogen elemental abundance ratio (Langer et al. 2016). The [C II] intensity from the neutral gas, $I_{\text {neutral }}([\mathrm{C}$ II $]$ ), is just the difference of the observed total $[\mathrm{C}$ II $]$ intensity, $I_{\text {tot }}([\mathrm{C} \mathrm{II}])$, minus the predicted $I_{\text {ion }}([\mathrm{C} \mathrm{II}])$ derived from $[\mathrm{NII}]$. We calculated the fraction of emission of [C II] from the ionized gas along $\left(0^{\circ}, 0^{\circ}\right)$ using the spectra shown in Fig. 6. However, to derive just the contribution from the $\mathrm{CMZ}$ we have to exclude the emission from the 3-kpc arm which dominates the total [N II] intensity. We find that the predicted $I_{\text {ion }}([\mathrm{C} \mathrm{II}])$ derived from [N II] is about $30 \%$ of the total $[\mathrm{C} \mathrm{II}]$ emission, $I_{\text {tot }}([\mathrm{C} \mathrm{II}])$, as derived from the [C II] spectrum, and the remaining $70 \%$ comes from PDRs and CO-dark $\mathrm{H}_{2}$ regions. This result holds only for this one path through the CMZ because we do not have [N II] spectra along the remaining positions in the two strip scans.

\section{Discussion}

In this section we discuss the properties of the following $\mathrm{CMZ}$ features traced by or near to the [C II] OTF strip scans: the open orbit gas streams, the Brick, the Radio Arc, the base of the arched filament, the warm dust bubble, Sgr B2, and an H II region likely associated with the local source S17.

\subsection{Open orbit gas streams}

Molinari et al. (2011) found that Herschel far-IR images of the CMZ revealed cold dense gas tracing out a roughly $200 \mathrm{pc}$ size $\infty$-shaped image, as seen in projection, centered slightly below $(l, b)=\left(0^{\circ}, 0^{\circ}\right)$. This feature corresponds well with the bowlike streams seen in CS by Tsuboi et al. (1999). Molinari et al. (2011) used the CS(1-0) spectral line maps and, assuming that this line traces dense cores in the clouds forming this feature, they assigned average velocities to the points along the $\infty$-shape. They proposed that the shape and velocity structure could be modeled roughly as an orbiting elliptical ring with an oscillating warp in the $z$-direction. They made a best fit to the CS data and found an ellipse with major and minor axes of $\sim 100 \mathrm{pc}$ and $\sim 60 \mathrm{pc}$, respectively, projected on the plane of the Galaxy (see their Fig. 5), an orbital velocity of $80 \mathrm{~km} \mathrm{~s}^{-1}$ (for simplicity assumed constant), and that the minor axis is oriented $40^{\circ}$ with respect to the line of sight to the Sun. The ellipse is assumed to lie $15 \mathrm{pc}$ below the plane and to have a vertical frequency twice the orbital frequency. The model parameters, as noted by Molinari et al. (2011), need to be used with caution given the 

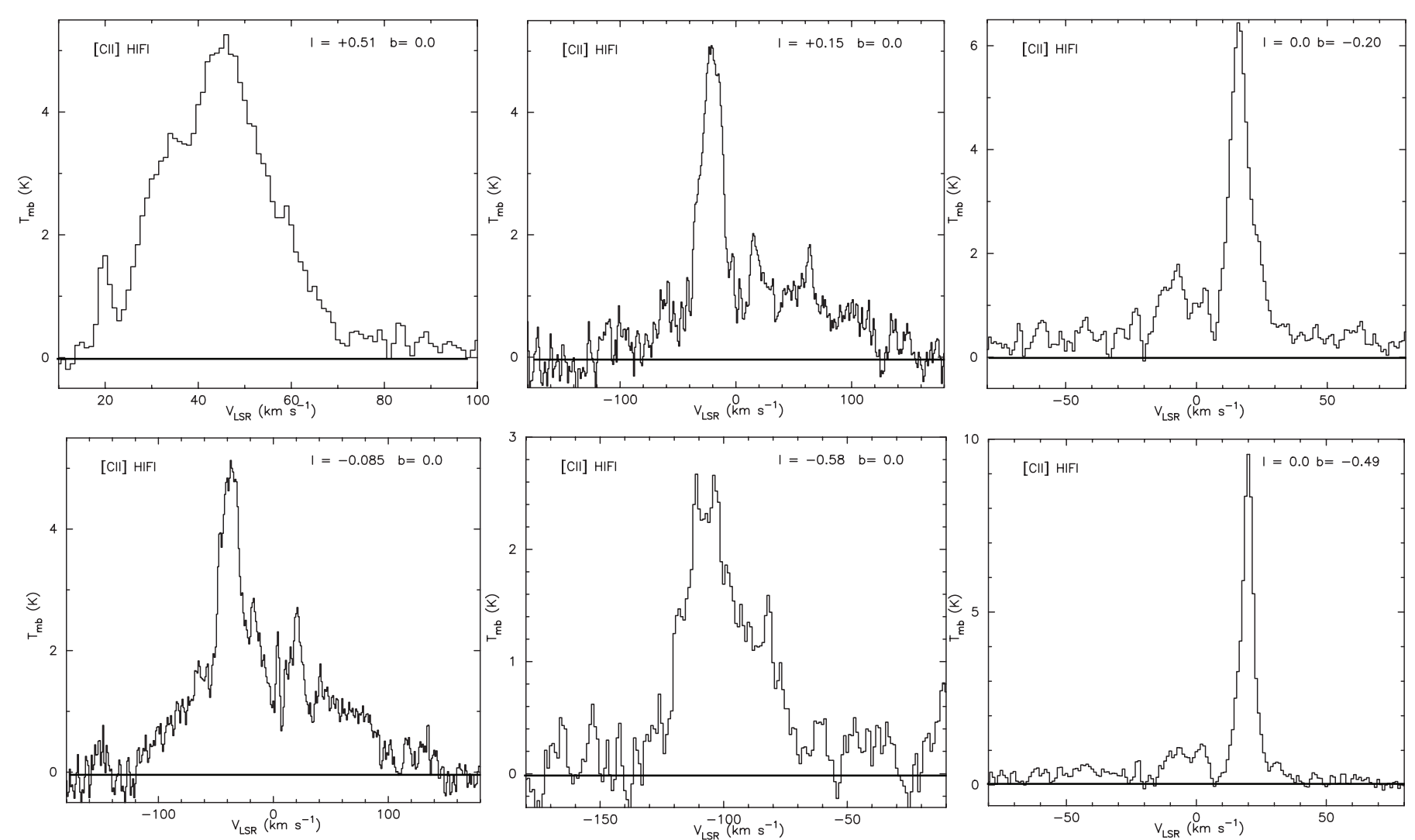

Fig. 5. GOT C $+[\mathrm{C}$ II] OTF spectra at six positions where $(0.51,0 \circ 0)$ and $(-0.58,0.0)$ are at the edges of the open orbiting gas stream, $(-0.085,0.0)$ is near Sgr A, and $(0.0,-0 \circ 20)$ and $(0 \circ 0,-0.49)$ show the narrowing of the [C II] emission below the plane.

oversimplification of the model and that the assigned $\mathrm{CS}(1-0)$ velocities are derived by eye. Nonetheless, the observed velocities are within $\pm 25 \mathrm{~km} \mathrm{~s}^{-1}$ of the model velocities.

However, as pointed out by Kruijssen et al. (2015) it is physically impossible to have a closed orbit in an extended gravitational potential. Instead they have replaced the singleorbit elliptical model of Molinari et al. (2011) by considering gas streams moving in an open orbit in the gravitational potential of the CMZ. Henshaw et al. $(2016 a, b)$ have expanded on this model. They find a best fit to the observed velocity field if they assume four streams (see Fig. 2 in Henshaw et al. 2016b). Furthermore, their orbital velocities vary along the orbit and are larger (up to $100-200 \mathrm{~km} \mathrm{~s}^{-1}$ ), than those in the elliptical ring model. Their best fit yields major and major and minor axes 121 and $59 \mathrm{pc}$, respectively, and an orbital velocity at apocenter and pericenter of 101 and $207 \mathrm{~km} \mathrm{~s}^{-1}$, respectively.

To see whether the $[\mathrm{CII}] l-V$ data encompasses the open orbit gas streams we derived the [C II] velocities as a function of longitude for $b=0^{\circ}$ using the elliptical model of Molinari et al. (2011) to find the envelope of these features in [C II]. This elliptical model also allows us to constrain the limits on orbital velocity. (Note that our use of the Molinari et al. (2011) model to define the approximate $(l, b, V)$ extent of dense molecular gas does not imply that the gas is on closed orbits.) The solid red line ellipse in Fig. 2a denotes the elliptical ring in longitude-velocity from Molinari et al. (2011) calculated for $b=0^{\circ}$. Our fit to the $[\mathrm{C} \mathrm{II}]$ data is shown as a red-dashed ellipse and, in order to accommodate the emission at $l= \pm 0.6$, requires an increase in orbital velocity to $\sim 100 \mathrm{~km} \mathrm{~s}^{-1}$ and orbit size of the major-axis to about $120 \mathrm{pc}$, as well as a slight change in the minor axis orientation to the line of sight to the

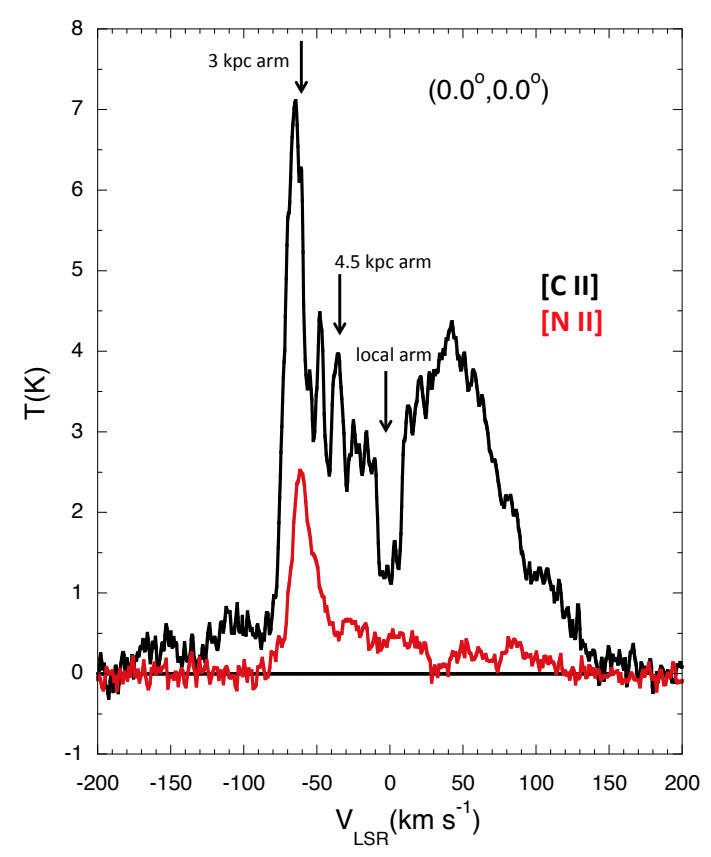

Fig. 6. Main beam temperature versus velocity for [C II] (black) and [N II] (red) spectra towards $\left(0^{\circ}, 0^{\circ}\right)$ taken with HIFI (Langer et al. 2016). The velocities of the foreground spiral arms are labeled.

sun (see Fig. 5 Molinari et al. 2011) to $\sim 50^{\circ}$. In general the [C II] emission follows the trend in the elliptical model, but the overall extent in longitude in [C II] is larger than that derived by Molinari et al. (2011) from CS(1-0), as is evident at the limits 

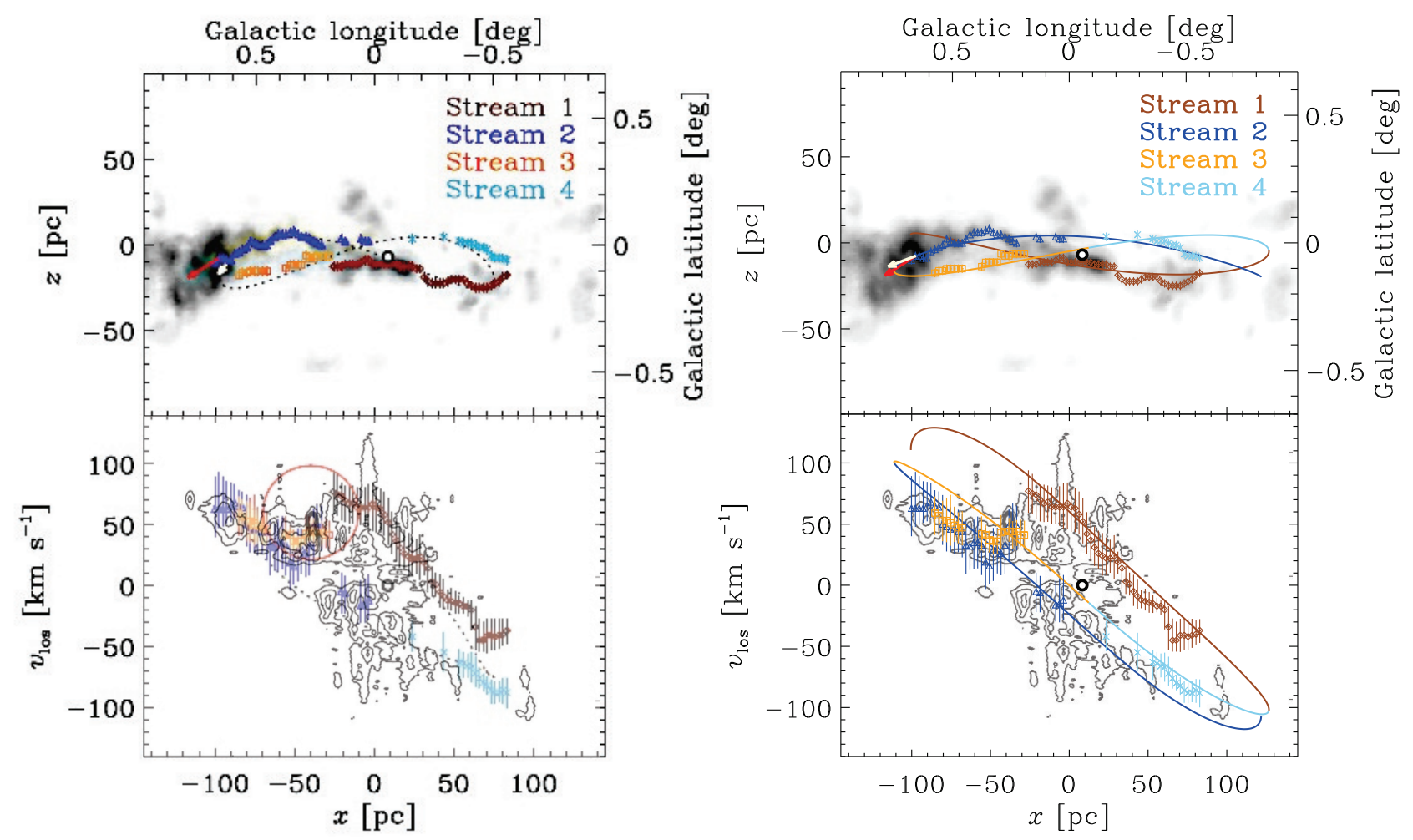

Fig. 7. GOT C+ [C II] (l-V) map compared with the Molinari et al. (2011) orbital model and with the four open stream orbital model of Kruijssen et al. (2015). Left panels: (l-b) and (l-V) maps in which the dotted line indicates the Molinari et al. (2011) orbital model (reproduced from Fig. 2 in Kruijssen et al. 2015). The grey-scale in the top panel is the integrated intensity of $\mathrm{NH}_{3}(1,1)$ emission that traces the dense gas. The symbols with error bars represent the phase-space information extracted by Kruijssen et al. (2015) from $\mathrm{NH}_{3}(1,1)$. The small open black circle denotes Sgr A*. (The circle in the left panel is in the original figure and indicates a feature in position-velocity space discussed by Kruijssen et al. 2015.) Right panels: $(l-b)$ and $(l-V)$ maps in which the solid open arcs indicate the four open gas streams and the legend labels them by color (reproduced from Fig. 4 in Kruijssen et al. 2015). The GOT C+ OTF scan at $b=0^{\circ}$ passes through streams 2 and 4, and passes closest to stream 3 near $l \sim 0.2$ and to stream 4 near $l \sim 0.05$. In the bottom panel the GOT C+ [C II] longitudinal-velocity contour plots are overlayed on the $l-V$ plots of the four stream open orbit model from Kruijssen et al. (2015).

of the ellipse at $l \simeq \pm 0$.6. In summary, the orbital velocity derived by Molinari et al. (2011) is too small to accommodate all the $[\mathrm{CII}]$ emission.

Our elliptical fit to the [C II] emission is in better agreement with the Kruijssen et al. (2015) and Henshaw et al. (2016b) open orbit gas stream models. To get a clearer picture of the relationship of [C II] emission to the open orbit gas streams, we compare the GOT C+ [C II] $(l-V)$ map with the with the four open stream orbital model of Kruijssen et al. (2015) and the Molinari et al. (2011) elliptical model. Figure 7 shows the $(l-b)$ and $(l-V)$ maps in Kruijssen et al. (2015) reproduced from their Fig. 2 (left panel in Fig. 7) and Fig. 4 (right panel in Fig. 7). The dotted line in the left panel indicates the Molinari et al. (2011) elliptical orbital model. The solid open arcs in the right panel show the four open gas streams and the legend labels them by color. The grey-scale in the top panels is the integrated intensity of $\mathrm{NH}_{3}(1,1)$ emission that traces the dense gas. The symbols with error bars represent the phase-space information extracted by Kruijssen et al. (2015) from $\mathrm{NH}_{3}(1,1)$ - see their paper for details. The GOT C+ OTF scan at $b=0^{\circ}$ passes through streams 2 and 4 , and passes closest to stream 3 near $l \sim 0.2$ and to stream 4 near $l \sim 0.05$.

In the bottom panel the GOT C+ [C II] longitudinal-velocity contour plots are overlayed on the $l-V$ plots of the four stream open orbit model from Kruijssen et al. (2015). The [C II] $(l-V)$ maps overlap both the elliptical and open stream models, but, as discussed above, the outermost $(l-b)$ [C II] emission fits better with the open stream model. The [C II] $l$ - $V$ emission correlates well with streams 1 and 2, and a portion of stream 3, while the correlation with stream 4 is unclear due to the weakness of the [C II] and overlap with stream 2. However, the association of [C II] emission with the dense gas in streams 2 and 3 is perhaps not surprising as both of them pass through the [C II] scan at $b=0^{\circ}$, however the association with the dense gas in stream 1 (and perhaps stream 4), which pass a few pc below the [C II] strip scan, suggests that there is lower density gas in a PDR, COdark $\mathrm{H}_{2}$, or ionized region associated with the dense gas streams. Longmore et al. (2013) and Henshaw et al. (2016b) propose that the gas along a stream represents a time sequence in the formation and evolution of star-forming dense clouds. If there is a time sequence then perhaps some of the [C II] in the $(l-V)$ map is tracing the $\mathrm{CO}$-dark $\mathrm{H}_{2}$ phase in early cloud formation or a late stage where it is being stripped away. If so then maps of the [C II] emission may be able to tell us something about the dynamics of the formation of the clouds in the gas streams.

One of the more interesting aspects of the orbiting gas streams is the presence of a corrugated velocity field with several regularly spaced features detected over $l \approx-0.1$ to -0.7 (Henshaw et al. 2016a), whose velocity maxima correlate with massive and compact clouds. Henshaw et al. (2016a) suggest that these features illustrate the mechanism of cloud formation in the inner $\mathrm{CMZ}$ and, in particular, highlight the regions where gas flow "traffic jams" seed cloud formation. The evidence for the ripples and condensations is derived from high density gas tracers such as $\mathrm{N}_{2} \mathrm{H}^{+}$, but direct evidence for the flows could 
come from spectral probes that trace the lower density outlying gas surrounding the cores. The [C II] longitudinal strip scan also cuts across or passes close to most of the region in which Henshaw et al. (2016a) detect the ripples in velocity.

To examine the relationship of [C II] emission to the corrugated velocity field we show in Fig. 8 a blow-up of the [C II] and $\mathrm{CO}(4-3) l-V$ maps in Fig. 4 (bottom-left panel) covering the region studied in Henshaw et al. (2016a) in $\mathrm{N}_{2} \mathrm{H}^{+}$. The region extends over $l=-0.7$ to 0.0 and $V_{\mathrm{LSR}}=-130 \mathrm{~km} \mathrm{~s}^{-1}$ to $-40 \mathrm{~km} \mathrm{~s}^{-1}$. For comparison to the dense gas tracers we overlay our [C II] $l-V$ maps on Fig. 2b from Henshaw et al. (2016a), which is a longitude-velocity maps of the centroid velocities of the $\mathrm{N}_{2} \mathrm{H}^{+}$spectral components. The $\mathrm{N}_{2} \mathrm{H}^{+} l-V$ plot lies close to several [C II] local peaks that appear to have a corrugated velocity structure in $l-V$ space. In addition, the $\mathrm{CO}(4-3)$ color image and $[\mathrm{CI}$ ] color image (not shown) appear to follow the gas streams. Thus the [C II], [C I] and $\mathrm{CO}(4-3)$ emissions are tracing the gas clouds in the open orbiting stream in this region. This relationship is to be expected, as the neutral $\mathrm{C}$ and $\mathrm{CO}$ layers lie close to, and in the case of $\mathrm{CO}$, overlap the dense cores in cloud chemical models, and [C II] lies in the associated PDRs, CO-dark $\mathrm{H}_{2}$ gas clouds, and ionized boundary layers.

The widespread distribution of [C II] as revealed by the large distribution in velocity and the blending of [C II] components makes it difficult to tell whether there is a systematic shift in velocity among the diffuse and dense gas tracers. Only spectrally resolved $l-b$ maps of [C II] of the orbiting gas stream can reveal whether there is evidence for the dynamical flows responsible for cloud formation in this region. To derive the absolute relationship between the [C II] velocity field and that of $\mathrm{N}_{2} \mathrm{H}^{+}$is beyond the scope of this paper, given the limits inherent in the longitudinal strip scan and the blending of many features in each [C II] spectrum.

\section{2. $\mathrm{G} 0.253+0.016$ (the Brick)}

G0.253+0.016, also known as the Brick, is an infrared-dark cloud at $l \sim 0.25$ centered slightly above $b=0^{\circ}$. There is little evidence of massive star formation in the Brick despite its high mass, $\sim(1-2) \times 10^{5} M_{\odot}$, and high local and average densities, $>10^{5}$ and $>10^{4} \mathrm{~cm}^{-3}$, respectively (Lis et al. 1994; Longmore et al. 2012; Rathborne et al. 2014; Mills et al. 2015). The detection of one $\mathrm{H}_{2} \mathrm{O}$ maser and several compact radio sources is consistent with the formation of perhaps at most a few early B-stars (Longmore et al. 2014; Mills et al. 2015). Pillai et al. (2015) derive the orientation and strength of the magnetic field in G0.253+0.016 from dust polarization maps. They find that the magnetic field is highly ordered and lies in an arch along the length of G0.253+0.016 and is sufficiently strong to support the cloud against collapse. Longmore et al. (2013) suggest that this cloud was tidally compressed perpendicular to its orbit and stretched along its orbit after passing through pericentre at its minimum in the Galactic gravitational potential. The [C II] emission towards the Brick is strong; in fact it has some of the brightest peaks in the CMZ strip scans, as can be seen in Figs. $2 \mathrm{a}$ and 4.

G0.253+0.016 has been well studied by Rathborne et al. (2014) using a suite of optically thin and thick spectral line map data of high density gas tracers, and dust continuum emission in the mid-infrared and $450 \mu \mathrm{m}$. In Fig. 9d we show the integrated HNCO intensity distribution from Fig. 6 in Rathborne et al. (2014) that traces out the dense core and the velocity field along the Brick. Our [C II] longitudinal strip scan is offset from the main core of the Brick as indicated by the horizontal red line

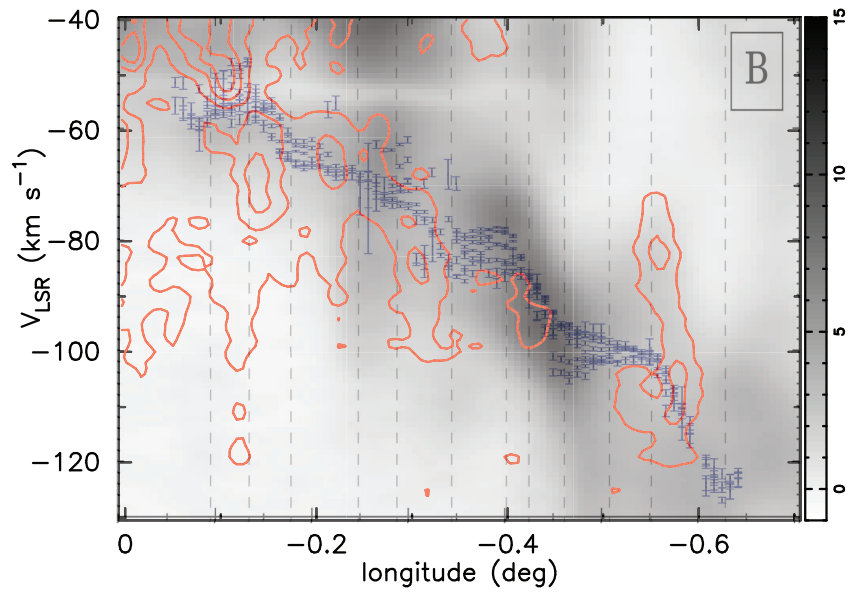

Fig. 8. Expanded view of the $l-V$ maps in Fig. 4 consisting of [C II] contours (red) and the $\mathrm{CO}(4-3)$ grey-scale image covering $l=-0.7$ to 0.0 and $V_{\mathrm{LSR}}=-130 \mathrm{~km} \mathrm{~s}^{-1}$ to $-40 \mathrm{~km} \mathrm{~s}^{-1}$. The [C II] coutours are $T_{\mathrm{mb}}=1,2,3,4,5$, and $6 \mathrm{~K}$, and the $\mathrm{CO}(4-3) T_{\mathrm{mb}}$ values are shown in the color bar on the right. We have superimposed the centroid velocities of the dense cores presented in Fig. $2 b$ from the paper by Henshaw et al. (2016a). (The vertical dashed lines are in the original figure from Henshaw et al. 2016a and identify the correspondence between the gas condensations and the extremes in velocity field amplitude.) It can be seen that the $l-V$ trends of $[\mathrm{C} \mathrm{II}]$ and $\mathrm{CO}(4-3)$ correlate well with those of the dense cores traced by $\mathrm{N}_{2} \mathrm{H}^{+}$.

at $b=0^{\circ}$. It can be seen that the [C II] spectral line data presented here pass through an edge of the Brick where $V_{\mathrm{LSR}} \sim 30$ to $40 \mathrm{~km} \mathrm{~s}^{-1}$. In contrast to the high dipole molecules, $\mathrm{CO},[\mathrm{C} \mathrm{I}]$, and $[\mathrm{C} \mathrm{II}]$ trace the less dense outer envelopes of G0.253+0.016, and probes an important spatial regime where dynamical effects (such as gas compression and stripping) due the orbital streaming motion of the clouds with respect to Galactic center are likely to be pronounced. Here we present the results of the [C II] emission and velocity structure and an analysis of the $\mathrm{H}_{2}$ molecular gas seen by [C II], [C I], and CO emissions.

In Fig. 9 we compare the [C II] $l$ - $V$ scans from $l=0.1$ to 0.4 at $b=0.0$, to three other gas tracers: $\mathrm{CO}(4-3),[\mathrm{C} \mathrm{I}]$, and $\mathrm{HN}^{13} \mathrm{C}$. Figure 9 a shows the $[\mathrm{C} \mathrm{II}]$ contours on top of a color image of the AST/RO CO(4-3) emission (Martin et al. 2004), where the $\mathrm{CO}(4-3)$ emission associated with $\mathrm{G} 0.253+0.016$ is strongest at $l \sim 0.25$ over a velocity range $V_{\mathrm{LSR}} \sim 20$ to $40 \mathrm{~km} \mathrm{~s}^{-1}$. The $\mathrm{CO}(4-3)$ emission over $l \sim 0.1$ to 0.3 at velocities 50 to $80 \mathrm{~km} \mathrm{~s}^{-1}$ is not associated with G0.253+0.016 and likely lies far from it (see footnote 15 in Rathborne et al. 2014). In addition, there are two very bright [C II] features that peak at $l \sim 0.21$.

Figure $9 \mathrm{c}$ shows the $[\mathrm{C} \mathrm{II}] l-V$ emission contours plotted over a color image of the $[\mathrm{CI}]$ emission. $[\mathrm{CI}]$ is much weaker than $\mathrm{CO}(4-3)$ across the Brick and is strongest at $l \sim 0.32$, near the edge of the $[\mathrm{C} \mathrm{II}]$, and weak at $\sim 0.21$, where $[\mathrm{C} \mathrm{II}]$ is stronger. The $\left[\mathrm{C}\right.$ II] peak at $V_{\mathrm{LSR}} \sim 30 \mathrm{~km} \mathrm{~s}^{-1}$ likely originates from the Brick's PDR or an H II region.

Figure $9 \mathrm{~b}$ shows the $[\mathrm{C} \mathrm{II}] l-V$ emission contours plotted over a color image of the $\mathrm{HN}^{13} \mathrm{C}$ emission. The $\mathrm{HN}^{13} \mathrm{C}$ data are from the Mopra ${ }^{3}$ survey (Jones et al. 2012). The $\mathrm{HN}^{13} \mathrm{C}$ emission, while much weaker than $[\mathrm{CII}],[\mathrm{CI}]$, and $\mathrm{CO}(4-3)$, peaks at

\footnotetext{
The data are from the Mopra radio telescope, a part of the Australia Telescope National Facility which is funded by the Commonwealth of Australia for operation as a National Facility managed by CSIRO. The University of New South Wales (UNSW) digital filter bank (the UNSW-MOPS) used for the observations with Mopra was provided with support from the Australian Research Council (ARC), UNSW, Sydney and Monash Universities, as well as the CSIRO.
} 

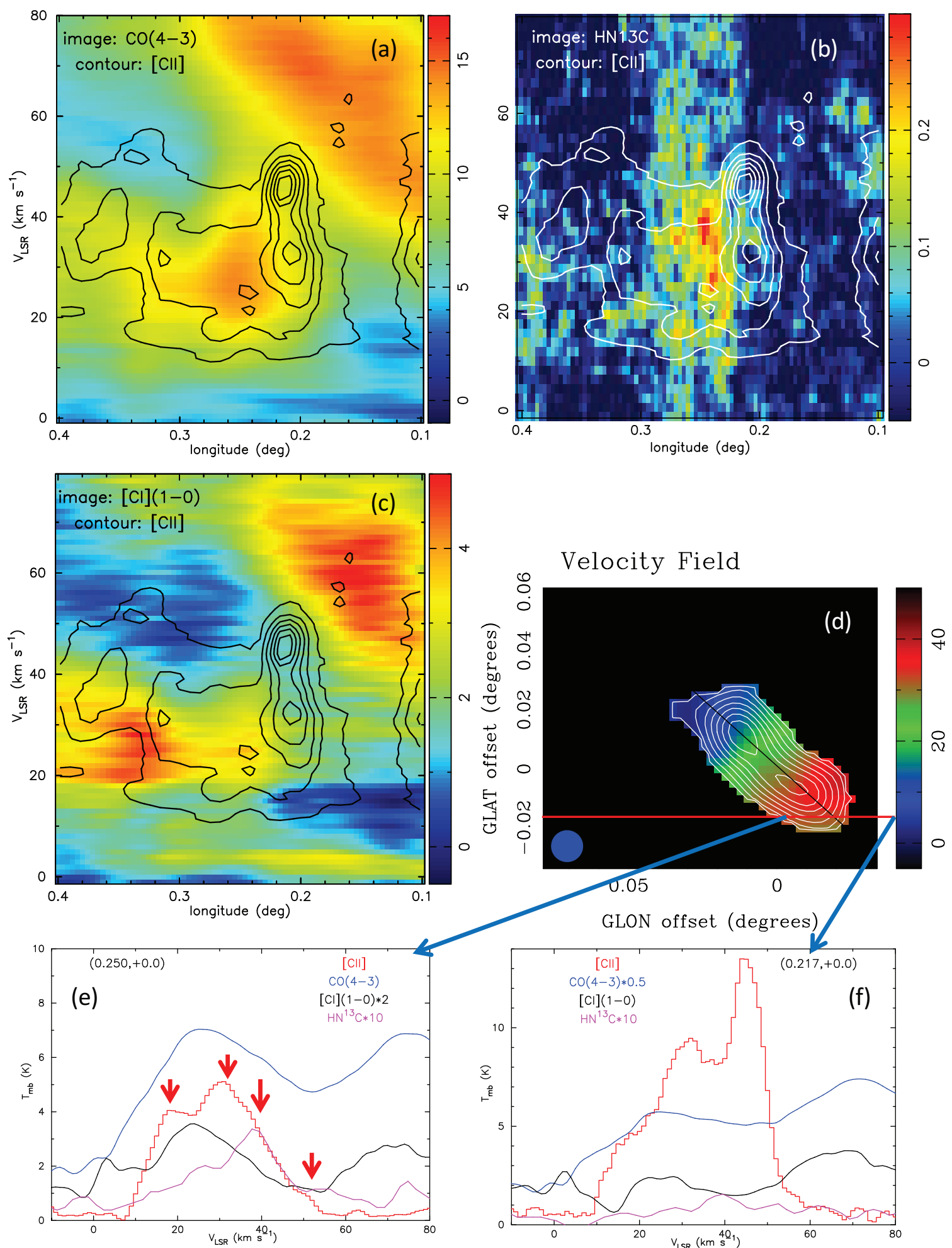

Fig. 9. Detailed view of the [C II] longitude-velocity contour plot of $T_{\mathrm{mb}}(\mathrm{K})$ associated with the Brick plotted over a map of a) CO(4-3) emission (Martin et al. 2004); b) $\mathrm{HN}^{13} \mathrm{C}$ Mopra data (this paper); and c) [C I] emission (Martin et al. 2004). The color bars give the values of $T_{\mathrm{mb}}(\mathrm{K})$ in the images; [C II] contour levels are 2, 4, 6, 8, and $10 \mathrm{~K}$, and the peak is $13.5 \mathrm{~K}$; d) the velocity field and HNCO intensity of the Brick from Fig. 6 in Rathborne et al. (2014) where the color bar on the right is the velocity field in $\mathrm{km} \mathrm{s}^{-1}$ and the intensity is shown by the contours. This plot is in offsets from the nominal center of the Brick, $(l, b)=(0.253,0.016)$. The horizontal red line designates $b=0^{\circ}$ and the blue arrows indicate the locations of the spectra shown in panels e) and f) which compare the GOT C+ [C II] with the AST/RO [C I] and CO(4-3) spectra (Martin et al. 2004), and the Mopra $\mathrm{HN}^{13} \mathrm{C}$ spectra (this paper) at $l=0.250$ and 0.21667 , respectively. The red arrows in e) indicate the four velocity components identified by Henshaw et al. (2016b) in the vicinity of G0.253+0.016 at $V_{\mathrm{LSR}} \sim 7,21,38$, and $70 \mathrm{~km} \mathrm{~s}^{-1}$ from dense gas tracers. The $\mathrm{HN}{ }^{13} \mathrm{C}$ peak at $\sim 38.4 \mathrm{~km} \mathrm{~s}^{-1}$ is the feature arising from the Brick at this position. The intensities are given as $T_{\mathrm{mb}}(\mathrm{K})$. 
Table 2. Model column densities for G0.250+0.00 for velocity component with $V_{\mathrm{LSR}}=38 \mathrm{~km} \mathrm{~s}^{-1}$.

\begin{tabular}{llccccccccc}
\hline \hline Species & Tracer & $\begin{array}{c}T_{\text {peak }}{ }^{a} \\
(\mathrm{~K})\end{array}$ & $\begin{array}{c}V_{\mathrm{LSR}} \\
\left(\mathrm{km} \mathrm{s}^{-1}\right)\end{array}$ & $\begin{array}{c}F W H M \\
\left(\mathrm{~km} \mathrm{~s}^{-1}\right)\end{array}$ & $\begin{array}{c}n\left(\mathrm{H}_{2}\right)^{b} \\
\left(\mathrm{~cm}^{-3}\right)\end{array}$ & $\begin{array}{c}T_{\text {kin }}{ }^{b} \\
(\mathrm{~K})\end{array}$ & $\tau^{c}$ & $\begin{array}{c}N(\mathrm{X}) \\
\left(\mathrm{cm}^{-2}\right)\end{array}$ & $\begin{array}{c}N\left(\mathrm{H}_{2}\right) \\
\left(\mathrm{cm}^{-2}\right)\end{array}$ & $\begin{array}{c}L \\
(\mathrm{pc})\end{array}$ \\
\hline $\mathrm{C}^{+}$ & {$[\mathrm{C} \mathrm{II}]^{2} \mathrm{P}_{3 / 2} \rightarrow{ }^{2} \mathrm{P}_{1 / 2}$} & 2.2 & 37.6 & 11.5 & $1 \times 10^{3}$ & 100 & 0.34 & $7.2 \times 10^{17}$ & $2.6 \times 10^{21}$ & 0.83 \\
$\mathrm{C}$ & {$[\mathrm{C} \mathrm{I}]^{2} \mathrm{P}_{1} \rightarrow{ }^{2} \mathrm{P}_{0}$} & 1.6 & 37.4 & 11.6 & $2 \times 10^{3}$ & 100 & 0.02 & $2.5 \times 10^{17}$ & $0.9 \times 10^{21}$ & 0.15 \\
$\mathrm{CO}$ & $\mathrm{CO}(4-3)$ & 10.0 & 38.2 & 11.6 & $2 \times 10^{3}$ & 70 & 6.3 & $6.0 \times 10^{17}$ & $2.1 \times 10^{21}$ & 0.35 \\
$\mathrm{HN}^{13} \mathrm{C}$ & $\mathrm{HN}^{13} \mathrm{C}(1-0)$ & 0.29 & 38.4 & 10.5 & $2 \times 10^{4}$ & 65 & 0.65 & $1.7 \times 10^{13}$ & $1.1 \times 10^{23}$ & 1.8 \\
\hline
\end{tabular}

Notes. ${ }^{(a)}$ Peak main beam temperature. ${ }^{(b)}$ The densities and kinetic temperatures are inputs to the radiative transfer model. ${ }^{(c)}$ Line opacity.

$l \sim 0.24$ and $V_{\mathrm{LSR}} \sim 38 \mathrm{~km} \mathrm{~s}^{-1}$. It falls off sharply at $l \sim 0.22$ and this boundary marks the transition from the dense core to the outer envelope traced by [C II]. Note also that the peak in $\mathrm{HN}^{13} \mathrm{C}$ intensity is distributed over $V_{\mathrm{LSR}} \sim 35$ to $45 \mathrm{~km} \mathrm{~s}^{-1}$, whereas the adjacent $[\mathrm{C}$ II] has a minimum.

There are two positions that we discuss in more detail, one is at $(l, b)=(0.250,0.0)$, hereafter G0.250+0.0, which is close to the nominal center of the dense core, and the other $(l, b)=$ $(0.217,0.0)$, hereafter $\mathrm{G} 0.217+0.0$, is outside the edge of the dense core where [C II] peaks. Here we discuss G0.250+0.0 and defer discussion of $\mathrm{G} 0.217+0.0$ to Sect. 4.3. Figure 9e shows the $[\mathrm{CII}],[\mathrm{CI}], \mathrm{CO}(4-3)$, and $\mathrm{HN}^{13} \mathrm{C}$ spectra at $l=0.25$, which is about $2.5 \mathrm{pc}$ from the region associated with the nominal center of G0.253+0.016, and which is also the source of strong emission in the dense gas tracers. It can be seen that there is emission by these tracers across the velocity range 10 to $50 \mathrm{~km} \mathrm{~s}^{-1}$. Henshaw et al. (2016b) has identified four velocity components in the region of G0.253+0.016 at $V_{\mathrm{LSR}} \sim 7,21$, 38 , and $70 \mathrm{~km} \mathrm{~s}^{-1}$, only one of which, at $38 \mathrm{~km} \mathrm{~s}^{-1}$, is associated with the Brick.

To understand better the velocity components in the spectra arising from $(l, b)=(0.250,0 \circ 0)$ we fit the line profiles with a multi-Gaussian function over the velocity range 0 to $60 \mathrm{~km} \mathrm{~s}^{-1}$, which more than covers the velocity range of the Brick. The two strongest Gaussian components for $\mathrm{HN}^{13} \mathrm{C}$ are at 26.0 and $38.4 \mathrm{~km} \mathrm{~s}^{-1}$, and the latter is clearly seen in Fig. 9e. The component at $38.4 \mathrm{~km} \mathrm{~s}^{-1}$ is associated with the Brick and agrees with that derived by Henshaw et al. (2016b). It is also closer to the $V_{\mathrm{LSR}} \sim 36.5 \mathrm{~km} \mathrm{~s}^{-1}$ derived by Rathborne et al. (2014) at their position P4 in the Brick (see their Fig. 7) which is about $1 \mathrm{pc}$ away from $(0.250,0 \circ 0)$. The line parameters of the fits to [C II], [C I], $\mathrm{CO}(4-3)$, and $\mathrm{HN}^{13} \mathrm{C}$ for the Brick component are given in Table 2. All four tracers have components associated with the Brick with $V_{\text {LSR }}$ within $1.0 \mathrm{~km} \mathrm{~s}^{-1}$ of each other, and full width half maximum (FWHM) linewidths $\sim 10.5$ to $11.6 \mathrm{~km} \mathrm{~s}^{-1}$ with $\mathrm{HN}^{13} \mathrm{C}$ being the narrowest line. The linewidths are in agreement with the intensity-weighted velocity dispersion derived by Rathborne et al. (2014) of $\sim 10$ to $15 \mathrm{~km} \mathrm{~s}^{-1}$.

In the open stream orbit models of Kruijssen et al. (2015) the core is compressed and collapses while the diffuse outer envelope is stripped away. The three carbon species $\mathrm{CO}, \mathrm{C}$, and $\mathrm{C}^{+}$occupy largely different layers in a cloud, progressing from $\mathrm{C}^{+}$on the outside to $\mathrm{CO}$ on the inside, with $\mathrm{C}$ mainly occupying a thin layer at the transition where all the main carbon species can coexist. In principle, one could derive the relative motion of the different gas layers from the velocity peaks of the [C II], [CI], $\mathrm{CO}(4-3)$, and $\mathrm{HN}^{13} \mathrm{C}$ to test the formation models for G0.253+0.016 (see discussion in Rathborne et al. 2014). Adopting $V_{\mathrm{LSR}} \sim 38.4 \mathrm{~km} \mathrm{~s}^{-1}$, the peak in $\mathrm{HN}^{13} \mathrm{C}$, as the velocity of the core, then the $\mathrm{C}^{+}$and $\mathrm{C}$ are blue shifted with respect to the core, which would be consistent with outflow of the envelope on the near side of the Brick, and infall on the far side. Because the $\mathrm{CO}(4-3)$ is optically thick it almost certainly arises from the near side of the core however the velocity difference of $0.2 \mathrm{~km} \mathrm{~s}^{-1}$ is well within the error of the fits and no conclusion can be drawn about any dynamical velocity difference. In addition, the blending of emission from many sources contributes to the spectra along this line of sight and even a difference of $\sim 1 \mathrm{~km} \mathrm{~s}^{-1}$ is within the uncertainties of a multi-Gaussian fit to lines that are significantly blended. To make further progress we need to have $l-b[\mathrm{C} \mathrm{II}]$ maps of this region to disentangle the velocity field.

To calculate the column densities of $\mathrm{CO}, \mathrm{C}, \mathrm{C}^{+}$, and $\mathrm{HN}^{13} \mathrm{C}$ we use the RADEX code (van der Tak et al. 2007), which is based on a large velocity gradient radiative transfer model, and the atomic and molecular data base of Schöier et al. (2005). Because $[\mathrm{C}$ II] has only one transition and we do not have spectra for the upper [CI] transition, ${ }^{2} \mathrm{P}_{2} \rightarrow{ }^{2} \mathrm{P}_{1}$, we need to assume a temperature and $\mathrm{H}_{2}$ density profile, as well as adopt a fractional abundance for carbon. The first two inputs are guided by existing information from other sources on the temperatures and densities of the cloud. The excitation results should be considered as a guide to possible conditions in the gas and not an exact solution.

We assume a kinetic temperature in the $\mathrm{CO}(4-3)$ layer of $70 \mathrm{~K}$, similar to the gas temperature of 65 to $70 \mathrm{~K}$ derived from $\mathrm{H}_{2} \mathrm{CO}$ (Ao et al. 2013), and $100 \mathrm{~K}$ in the $\mathrm{C}$ and $\mathrm{C}^{+}$layers, typical of the gas temperatures in the envelope (Clark et al. 2013). For $\mathrm{HN}^{13} \mathrm{C}$ we use the interior gas temperature of $65 \mathrm{~K}$. At these high kinetic temperatures the solutions are not that sensitive to uncertainties in the values chosen because they are comparable to or larger than the equivalent temperatures of the upper levels of $\mathrm{CO}(4-3),[\mathrm{CI}$, and [C II], which are 55.3, 23.6, and $91.2 \mathrm{~K}$, respectively. For the density profile we assume that the density in the $\mathrm{CO}(4-3)$ layer is much less than the average core density of $2 \times 10^{4} \mathrm{~cm}^{-3}$, and adopt $n\left(\mathrm{H}_{2}\right)=2 \times 10^{3} \mathrm{~cm}^{-3}$, which is much less than the critical density, $n_{\mathrm{cr}}\left(\mathrm{H}_{2}\right) \sim 4 \times 10^{4} \mathrm{~cm}^{-3}$, and thus the solutions are density sensitive. For the [CI] layer we also adopt $n\left(\mathrm{H}_{2}\right)=2 \times 10^{3} \mathrm{~cm}^{-3}$, which is larger than the critical density $\sim 1.1 \times 10^{3} \mathrm{~cm}^{-3}$ and thus the [C I] intensity is relatively insensitive to uncertainties in $\mathrm{H}_{2}$ density. In the [C II] layer we adopt $n\left(\mathrm{H}_{2}\right)=10^{3} \mathrm{~cm}^{-3}$ which is much less than the critical density $n_{\mathrm{cr}}\left(\mathrm{H}_{2}\right) \sim 4.5 \times 10^{3} \mathrm{~cm}^{-3}$. For the $\mathrm{HN}^{13} \mathrm{C}$ layer we adopt $2 \times 10^{4} \mathrm{~cm}^{-3}$.

To convert the column densities to a molecular $\mathrm{H}_{2}$ column density we adopt a fractional abundance of total carbon with respect to molecular hydrogen, $x\left(C_{\text {tot }}\right)=2.8 \times 10^{-4}$ as used in the models of Bertram et al. (2016). In most PDR models there is a very sharp transition from $\mathrm{C}^{+}$to $\mathrm{CO}$ as the $\mathrm{UV}$ radiation field is attenuated (cf. Bergin et al. 1997), while neutral carbon is dominant in a thin layer (Wolfire et al. 2010). For simplicity 
we assume that there is no mixing and the layers are either 100 percent ionized carbon, carbon monoxide, or neutral carbon, which is a reasonable approximation in the transition layer (see Wolfire et al. 2010). In the case of $\mathrm{HN}^{13} \mathrm{C}$ we adopt a fractional abundance of $3 \times 10^{-9}$ and an isotope ratio ${ }^{12} \mathrm{C} /{ }^{13} \mathrm{C}$ from Rathborne et al. (2014), which is consistent with cloud model fractional abundances of order $(1-6) \times 10^{-9}$ (Bergin et al. 1997).

The solutions are given in Table 2 where we list the assumed molecular hydrogen density, $n\left(\mathrm{H}_{2}\right)$, and kinetic temperature, $T_{\text {kin }}$, the solution to the column density of the tracer, $N(\mathrm{X})$, the total $\mathrm{H}_{2}$ column density, $N\left(\mathrm{H}_{2}\right)$, and the depth of the emission layer $L$ in pc. We also list the line opacity for each tracer and find that the $[\mathrm{CII}]$ and $[\mathrm{CI}]$ emissions are optically thin, while the $\mathrm{CO}(4-3)$ emission is optically thick. Because the $\mathrm{CO}(4-3)$ transition is optically thick and sensitive to the choice of density one must use this result with caution. It can be seen that the [C II] layer is the largest, extending over $0.8 \mathrm{pc}$, while the $\mathrm{C}$ layer is smallest, $0.15 \mathrm{pc}$, which is typical of PDR models. The neutral carbon column density is relatively insensitive to the choice of local density, $n\left(\mathrm{H}_{2}\right)$, owing to the insensitivity of the upper level population to density for densities $\geq$ the critical density. The derived thickness of the $\mathrm{CO}$ layer, which is only $0.35 \mathrm{pc}$, should be viewed with caution owing to uncertainty associated with the large opacity and sensitivity to choice of density.

The $\mathrm{HN}^{13} \mathrm{C}$ column density translates to $N(\mathrm{HNC}) \sim 3.4 \times$ $10^{14} \mathrm{~cm}^{-2}$ at the edge compared to $10^{15} \mathrm{~cm}^{-2}$ derived by Rathborne et al. (2014) through the densest part of the core. Our results at the edge along $b=0^{\circ}$ are consistent with the core values as the $\mathrm{HN}^{13} \mathrm{C}$ intensities at the edge are about $10-20 \%$ of those in the core. The column density of $\mathrm{HN}^{13} \mathrm{C}$ corresponds to a depth of $1.8 \mathrm{pc}$. The total depth of the Brick at this location, as traced by the four carbon species is $L \sim 3.1 \mathrm{pc}$, as compared to $5.8 \mathrm{pc}$, twice the effective radius of the Brick (Rathborne et al. 2014). Our estimate of the path length at the edge is consistent with the decrease expected from the core value.

Most significantly, the [C II] emission is tracing some portion of the CO-dark $\mathrm{H}_{2}$ gas surrounding the core. Therefore, it is likely that the [C II] emission is probing a thick layer of $\mathrm{H}_{2}$ without $\mathrm{CO}$. We cannot say whether this $\mathrm{CO}$-dark $\mathrm{H}_{2}$ gas is undergoing compression in a collapsing envelope or being stripped away from a region where $\mathrm{CO}$ was dissociated.

If the Brick is a site with little or no local star formation, especially lacking $\mathrm{O}$ stars, then the bright [C II] emission from its edge at $\sim 32 \mathrm{~km} \mathrm{~s}^{-1}$ requires some other external source of ionization. Mills et al. (2015) come to a similar conclusion from maps of widespread, but weak, radio continuum emission, tracing ionized gas near the Brick. Mills et al. (2015) suggest that an O4-6 supergiant about $11 \mathrm{pc}$ away (in projection) to the east is a plausible source of ionizing photons to explain the ionized gas giving rise to radio continuum emission. Another possibility is that X-ray ionization is the source of the high density hot ionized gas leading to intense [C II] via electron excitation. Surveys of X-ray emission in the CMZ with Chandra (Wang et al. 2002), XMM (Ponti et al. 2015), and Suzaku (Koyama et al. 2007), reveal a rich X-ray environment containing extended diffuse emission (Koyama et al. 2007), and over 9000 discrete sources (Muno et al. 2009). As pointed out by Porquet et al. (2003) there is also a bright discrete source of X-rays, 1E1743.1+2843, located at $l=0.26$ and $b \sim 0.03$, about $6 \mathrm{pc}$ in projection from the Brick. This source, which is the brightest Galactic center X-ray source detected by XMM-Newton (Ponti et al. 2015), has an inferred X-ray luminosity $\sim 2 \times 10^{36} \mathrm{erg} \mathrm{s}^{-1}$ above $2 \mathrm{keV}$ and is likely an accreting neutron star binary (Porquet et al. 2003). However, one needs to know the luminosity down to
0.5 or $1 \mathrm{keV}$ to evaluate its influence on the $[\mathrm{CII}]$ region (Langer \& Pineda 2015). Unfortunately, X-rays in this range are absorbed by high column densities of intervening material and not measured directly, so we do not know the total relevant X-ray luminosity.

\section{3. [C II] peak at $\mathrm{G} 0.217+0.0$}

The [C II] emission at $(l, b)=(0.21667,0.0)$, hereafter $\mathrm{G} 0.217+0.0$, has two of the strongest [C II] emission peaks found in our strip scans. It is located close to or outside the edge of the dense core of the Brick (Fig. 9b). In the position velocity maps in Fig. 9a and $\mathrm{c}$ this feature is located between one dense core (the Brick) and a less dense core (seen in [C I] and $\mathrm{CO}(4-3)$ at $V_{\mathrm{LSR}} \sim 50 \mathrm{~km} \mathrm{~s}^{-1}$ but not in $\mathrm{HN}^{13} \mathrm{C}$. It is a relatively compact spatial feature in $[\mathrm{C}$ II] that peaks at $l \sim 0.21$. In Fig. 9f we show the [C II], [CI], $\mathrm{CO}(4-3)$, and $\mathrm{HN}^{13} \mathrm{C}$ spectra at this position. The velocity profile for [C II] has two peaks, $T_{\mathrm{mb}} \simeq 13.5 \mathrm{~K}$ at $V_{\mathrm{lsr}} \sim 45 \mathrm{~km} \mathrm{~s}^{-1}$ and $T_{\mathrm{mb}} \simeq 9.5 \mathrm{~K}$ at $\sim 30 \mathrm{~km} \mathrm{~s}^{-1}$. CO(4-3) is generally stronger than [C II] at this location while $[\mathrm{C} \mathrm{I}]$ is much weaker. Neither $\mathrm{CO}(4-3)$ nor [C I] show clear evidence of a double peak in velocity within the range of the [C II] emission. The $\mathrm{HN}^{13} \mathrm{C}$ spectrum is very weak at these two positions, as might be expected outside the edge of the dense core.The multi-Gaussian fit to the $[\mathrm{C} \mathrm{II}]$ profile at this location yields the following line parameters for the two prominent velocity features: $V_{\mathrm{LSR}} \sim 31.1$ and $44.5 \mathrm{~km} \mathrm{~s}^{-1}$, with $T_{\mathrm{mb}} \sim 8.9$ and $12.9 \mathrm{~K}$, and $F W H M \sim 11.3$ and $10.0 \mathrm{~km} \mathrm{~s}^{-1}$, respectively. The integrated intensities for these features are $I([\mathrm{C} \mathrm{II}]) \simeq 101$ and $130 \mathrm{~K} \mathrm{~km} \mathrm{~s}^{-1}$. The strength of the [C II] line and its spatial distribution in longitude suggest that it likely arises from an ionized boundary layer or extended H II region. If we assume it comes entirely from the IBL then we can estimate the electron density in this region using an approach similar to that outlined in Velusamy et al. (2012). Here we outline an approach to estimate $n(e)$. The $\mathrm{C}^{+}$column density as a function of the $[\mathrm{C} \mathrm{II}]$ intensity in the optically thin limit can be written (Langer et al. 2015, Eq. (1)),

$N\left(\mathrm{C}^{+}\right)=2.92 \times 10^{15}\left[1+\frac{e^{\Delta E / k T}}{2}\left(1+\frac{n_{\mathrm{cr}}(e)}{n(e)}\right)\right] I([\mathrm{C} \mathrm{II}]) \mathrm{cm}^{-2}$

where $I([\mathrm{C} \mathrm{II}])$ is the intensity in $\mathrm{K} \mathrm{km} \mathrm{s}^{-1}, \Delta E / k=91.21 \mathrm{~K}$ is the energy needed to excite the ${ }^{2} \mathrm{P}_{3 / 2}$ level, $n(e)$ is the electron density, and $n_{\mathrm{cr}}(e)$ is the corresponding electron critical density. The collisional rate coefficient for electrons implies $n_{\mathrm{cr}}(e) \sim 50 \mathrm{~cm}^{-3}$ at $T_{\text {kin }}=8000 \mathrm{~K}$ (Goldsmith et al. 2012), typical for highly ionized gas.

If we assume that the density is uniform over the observed emission path length and that the gas is completely ionized then, $N\left(\mathrm{C}^{+}\right)=n\left(\mathrm{H}^{+}\right) x\left(\mathrm{C}^{+}\right) L$ where $x\left(\mathrm{C}^{+}\right)$is the fractional abundance of ionized carbon, and $L$ is the path length in cm. Substituting $n(e)=n\left(\mathrm{H}^{+}\right)$we have a quadratic equation relating $n(e)$ to $I([\mathrm{C}$ II $])$ and $L$,

$\frac{n(e)^{2}}{\left[n(e)+n_{\mathrm{cr}}(e) / 3\right]}=4.4 \times 10^{15} \frac{I([\mathrm{C} \mathrm{II}])}{x\left(C^{+}\right) L(\mathrm{~cm})} \mathrm{cm}^{-3}$

where we have set the factor $\exp (\Delta E / k T)$ equal to 1 , as $\Delta E / k T \ll 1$ in a hot ionized gas. If we know the size of the emission region, $L$, we can solve Eq. (2) for $n(e)$ (or equivalently $\left.n\left(\mathrm{H}^{+}\right)\right)$as a function of $I([\mathrm{C} \mathrm{II}])$ for a given $x\left(\mathrm{C}^{+}\right)$and $T_{\text {kin. The }}$ exact value of the kinetic temperature of the hot ionized gas is not critical to the solution because $n_{\mathrm{cr}}(e)$ is a weak function of kinetic temperature, $\propto T_{\text {kin }}^{0.37}$ (Goldsmith et al. 2012). 
To estimate a scale size for the [C II] emission region along the line of sight at $(0.217,0 \circ 0)$ we assume that it is roughly the size of the longitudinal width of the [C II] ridge in Fig. 9, $\sim 0.04$, which at the distance to the Galactic center is $\sim 5.8 \mathrm{pc}$. Assuming this emission comes from warm ionized gas with $T_{\mathrm{k}}=8000 \mathrm{~K}$ and a fractional abundance $x\left(\mathrm{C}^{+}\right)=2.8 \times 10^{-4}$, we find that $n(e) \sim 102 \mathrm{~cm}^{-3}$ and $128 \mathrm{~cm}^{-3}$ for the $V_{\mathrm{LSR}} \sim 31.1$ and $44.5 \mathrm{~km} \mathrm{~s}^{-1}$ features, respectively. (If the emitting layer is more like a 2D surface, then we have overestimated the path length and the density will be higher.) The density is consistent with those found from studies using the [N II] far-IR fine structure lines to determine $n(e)$ in dense bright $\mathrm{H}$ II regions (Oberst et al. 2011) and in Sgr A (Goldsmith et al. 2015), but two to five times higher than those possibly characteristic of the ionized boundary layers (IBL) of clouds in the disk (Goldsmith et al. 2015) and clouds at the edge of the CMZ (Langer et al. 2015).

\subsection{The radio arc region}

The radio arc region is a set of very prominent radio filament features near Sgr A consisting of the nonthermal filaments (NTF) of the Radio Arc, the thermal Radio Arches, and nonthermal Radio Threads, as can be seen in the left panel of Fig. 3, which is a radio continuum map presented in Simpson et al. (2007) and adapted from Yusef-Zadeh \& Morris (1987; see also Fig. 1 in Lang et al. 2001, 2002). The lines-of-sight of the GOT C+ longitudinal strip scan intercept the base of the arched filaments over $l \sim 0.04$ to $\sim 0.08$ and the NTF Radio Arc filaments from $l \sim 0.15$ to $\sim 0.18$.

In Fig. 10 we mark the velocities of peak emission in the H92 $\alpha$ spectrum at three locations from Fig. 7 in Lang et al. (2001) which are closest to that of the [C II] spectra for $l=0.06$ at $V_{\mathrm{LSR}}=-26 \mathrm{~km} \mathrm{~s}^{-1},-43 \mathrm{~km} \mathrm{~s}^{-1}$, and $-34 \mathrm{~km} \mathrm{~s}^{-1}$ (upper panel) in locations 15, 16, and 17, respectively, and for $l=0.16$ at $V_{\mathrm{LSR}}=-21 \mathrm{~km} \mathrm{~s}^{-1},-30 \mathrm{~km} \mathrm{~s}^{-1}$, and $-27 \mathrm{~km} \mathrm{~s}^{-1}$ (lower panel) in locations 1,2 , and 8 , respectively. Note that all these locations are offset from $b=0^{\circ}$ at slightly higher latitude ( $\sim 2$ to 3 arcmin) but their longitudes are within the effective beam size of the [C II] map. In the $8.3 \mathrm{GHz}$ continuum map (Fig. 7 in Lang et al. 2001) all the arches near $l=0.06$ appear to extend down towards $b=0^{\circ}$ where they seem to merge into a more diffuse structure. The horizontal double-arrows mark the $\mathrm{CS}(2-1)$ velocity range for molecular clouds in the vicinity of $l=0.06$ (Lang et al. 2002) and $l=0.16$ (Serabyn \& Guesten 1987). The broad [C II] feature at $V_{\mathrm{LSR}} \sim-70 \mathrm{~km} \mathrm{~s}^{-1}$ in the spectrum for $l=0.06$ is associated with the open orbit gas streams (see Sect. 4.1).

Detection of these velocity features in the [C II] spectra at $l=0.06$ suggests significant contribution to the $[\mathrm{C} \mathrm{II}]$ emission from the ionized gas in the arches. The close association of the two [C II] velocities with the $\mathrm{H} 92 \alpha$ spectra at locations 16 and 17 in Lang et al. (2001), and the separation from the CS(2-1) molecular cloud emission, indicates that this [C II] emission is likely tracing parts of the thermal H I filaments at $l=0.06$. The $8.3 \mathrm{GHz}$ continuum maps in this region along $b=0^{\circ}$ appear to trace the base of the arched thermal filaments, which are also seen in $[\mathrm{C} \mathrm{II}]$ at the velocities for the filaments. However, because the velocity of the feature at location 15 is within the range of the CS velocities we cannot tell whether it is coming from the base of this filament or the CS molecular cloud envelope.

The three filaments in Fig. 7 from Lang et al. (2001) for $l=$ 0.16 that correspond to the velocities marked in Fig. 10 (lower panel) seem less pronounced in the region observed in [C II]. Therefore, in contrast to the emission at $l \sim 0.06$, the bright [C II] emission at $l \sim 0.16$ in the velocity range $-5 \mathrm{~km} \mathrm{~s}^{-1}$ to $-45 \mathrm{~km} \mathrm{~s}^{-1}$ may arise from an extension of the negative-velocity

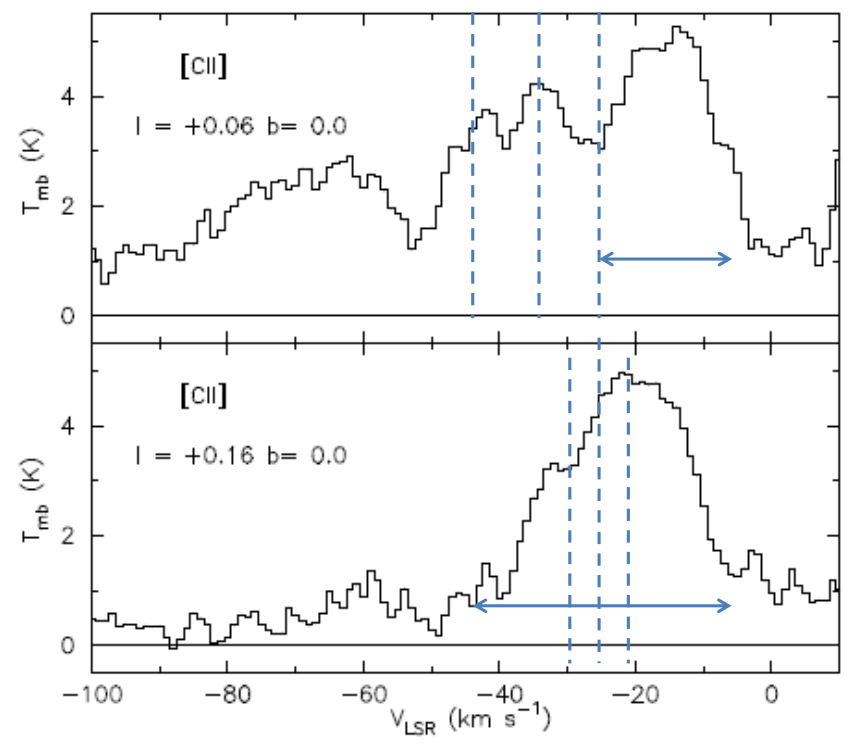

Fig. 10. $[\mathrm{CII}]$ spectra at two longitudes containing negative velocity clouds underlying the arched radio filaments (Lang et al. 2001). The dashed lines mark velocity features also observed in $\mathrm{H} 92 \alpha$ radio recombination lines associated with the thermal (ionized gas) component in the nearby radio arches. The horizontal double-arrows mark the $\mathrm{CS}(2-1)$ velocity ranges for molecular clouds in the vicinity of $l=0.06$ (Lang et al. 2002) and $l=0.16$ (Serabyn \& Guesten 1987).

molecular cloud underlying the arched filaments, which shows $\mathrm{CS}$ emission in the velocity range approximately $-50 \mathrm{~km} \mathrm{~s}^{-1}$ to $\sim 0 \mathrm{~km} \mathrm{~s}^{-1}$ (Serabyn \& Guesten 1987). The bright [C II] emission at $l \sim 0.15$ to 0.18 occurs right at the interface between the dense clouds seen in CS and the NTFs, where the thermal radio emission is relatively weak. In addition, there is evidence for [N II] emission from this region in the HEXGAL channel maps (see Appendix F of García 2015). Therefore, this emission might arise from a dense PDR and/or the ionized boundary layer associated with the CS molecular clouds.

At $l \sim 0.06$ the $[\mathrm{CII}]$ emission from the thermal arched filaments at $-34 \mathrm{~km} \mathrm{~s}^{-1}$ and $-43 \mathrm{~km} \mathrm{~s}^{-1}$ probably arises from electron excitation of $\mathrm{C}^{+}$. For these components we can use the $[\mathrm{CII}]$ emission to estimate the electron density associated with the base of the filaments following an approach similar to that for G0.217+0.0 in Sect. 4.3. We assume that the filaments are completely ionized and at a kinetic temperature of $8000 \mathrm{~K}$. (Lang et al. (2001) quote an ion temperature of $6900 \mathrm{~K}$, but the solutions to $n(e)$ are relatively insensitive to this kinetic temperature difference, as discussed in Sect. 4.2). The radio filaments in the $8.3 \mathrm{GHz}$ continuum maps near $(0.06,0.0)$ are about $50^{\prime \prime}$ wide or $\sim 2 \mathrm{pc}$ in width along the longitudinal strip scan. Thus the [C II] emission in each filament is slightly beam diluted along the scan direction by about a factor of 1.7. If we assume that the filament is cylindrically symmetric, then the path length of [C II] emission is the same as the width of the filament. We can use Eq. (2) to solve for the electron density. The [C II] components at $-34 \mathrm{~km} \mathrm{~s}^{-1}$ and $-43 \mathrm{~km} \mathrm{~s}^{-1}$ have an integrated intensity, $I([\mathrm{C} \mathrm{II}])$, of 47 and $35 \mathrm{~K} \mathrm{~km} \mathrm{~s}^{-1}$, respectively. Putting all these factors into Eq. (2) yields $n(e) \sim 100 \mathrm{~cm}^{-3}$ and $80 \mathrm{~cm}^{-3}$ at $-34 \mathrm{~km} \mathrm{~s}^{-1}$ and $-3 \mathrm{~km} \mathrm{~s}^{-1}$, respectively, at the base of the arched filaments. These densities are lower than the electron density derived using the $8.3 \mathrm{GHz}$ radio continuum intensity at peak $\mathrm{F}$ (Lang et al. 2001), $n(e) \sim 340 \mathrm{~cm}^{-3}$, located about $1^{\prime}$ away in $b$. The lower $n(e)$ at $b=0^{\circ}$ is consistent with the weaker and 
more diffuse emission in the $8.3 \mathrm{GHz}$ maps near the base of the thermal arched filaments.

\section{5. [C II] cloud emission models}

Recently Clark et al. (2013) and Bertram et al. (2016) presented numerical hydrodynamical simulations of CMZ clouds that include many physical processes, including thermal heating and cooling, chemical reaction networks, and radiative transfer models, with the goal of producing synthetic emission maps for comparison to observations. In particular, Bertram et al. (2016) produce velocity integrated intensity maps for [C II] (see their Fig. 4) for three different initial values of the ratio of the cloud's kinetic to potential energy, $\alpha=0.5,2.0$, and 8.0. The peak intensities in the spatial-spatial maps $I([\mathrm{C}$ II] $)$ are of order 50 to $100 \mathrm{~K} \mathrm{~km} \mathrm{~s}^{-1}$ regardless of $\alpha$, but the larger the value of $\alpha$ the larger the physical dimensions of the cloud. We cannot make a direct comparison of the GOT C+ observed [C II] intensities to the model maps because we only have strip scans. However, for the $[\mathrm{C} \mathrm{II}]$ integrated intensities and the size of the clouds intercepted in the longitudinal strip scans, excluding the region near Sgr A, are in the range 50 to $110 \mathrm{~K} \mathrm{~km} \mathrm{~s}^{-1}$, similar to the models in Bertram et al. (2016) $\alpha=0.5$.

\subsection{Warm dust bubble}

The warm dust bubble, as seen in the dust temperature image in Fig. 3 (Fig. 3 in Molinari et al. 2011), has the appearance in projection of a doughnut. Molinari et al. (2014) describe it as a very young superbubble with a roughly 15 pc radius containing the Arches and Quintuplet Clusters. The exact nature of the energy source powering this bubble remains unclear. The GOT C+ latitudinal strip scan passes through the ring of the warm dust doughnut while the longitudinal strip scan is about halfway between the upper and lower edge of the ring. In Fig. 3 we also plot the integrated $[\mathrm{C} \mathrm{II}]$ intensity, $I([\mathrm{C} \mathrm{II}])=\int T_{\mathrm{mb}} \mathrm{d} v\left(\mathrm{~K} \mathrm{~km} \mathrm{~s}^{-1}\right)$, as a function of longitude (bottom) and latitude (right side) for two different LSR velocity ranges: 80 to $130 \mathrm{~km} \mathrm{~s}^{-1}$ away (red) and -80 to $-130 \mathrm{~km} \mathrm{~s}^{-1}$ towards (blue) the sun, corresponding to the far and near sides of the bubble, respectively, assuming that the bubble is expanding. The strip scan in latitude shows a shift in $b$ in the peak [C II] emission between the blue- and red-shifted components. These two velocity ranges may be tracing the expansion of the gas associated with the dust bubble. Although it is also possible that the latitude offset between these two velocity regimes is attributable to the tilt in the CMZ (Morris \& Serabyn 1996, and references therein).

\subsection{Sgr B2}

Sgr B2 is one of the most massive molecular clouds in the Galaxy and a well-known star-forming region. The compact $\mathrm{H}$ II regions, Sgr B2(N) at $(l, b) \sim(0.6773,-0.02776)$ and Sgr B2(M) at $(l, b) \sim(0.6671,-0.0350)$ (Lis et al. 1994), are two very active star forming cores with a $V_{\mathrm{LSR}} \sim 60$ to $70 \mathrm{~km} \mathrm{~s}^{-1}$. They lie about 4 pc and 5 pc, respectively, below the [C II] longitudinal strip scan. The narrow beam size in latitude $\left(12^{\prime \prime}\right)$ in the [C II] $l-V$ map precludes any detection from the Sgr B2(N) and Sgr B2(M) cores themselves, however it does sample the gas that may be influenced by their stellar activity. As seen in Figs. $2 \mathrm{a}$ and 4 at $(l, b) \sim(0 \circ 67,0.0)$ there is strong $\mathrm{CO}(4-3)$ emission over a broad range in velocity, but [C II] emission is only over a narrow velocity range around $V_{\mathrm{LSR}} \sim 65 \mathrm{~km} \mathrm{~s}^{-1}$. In view

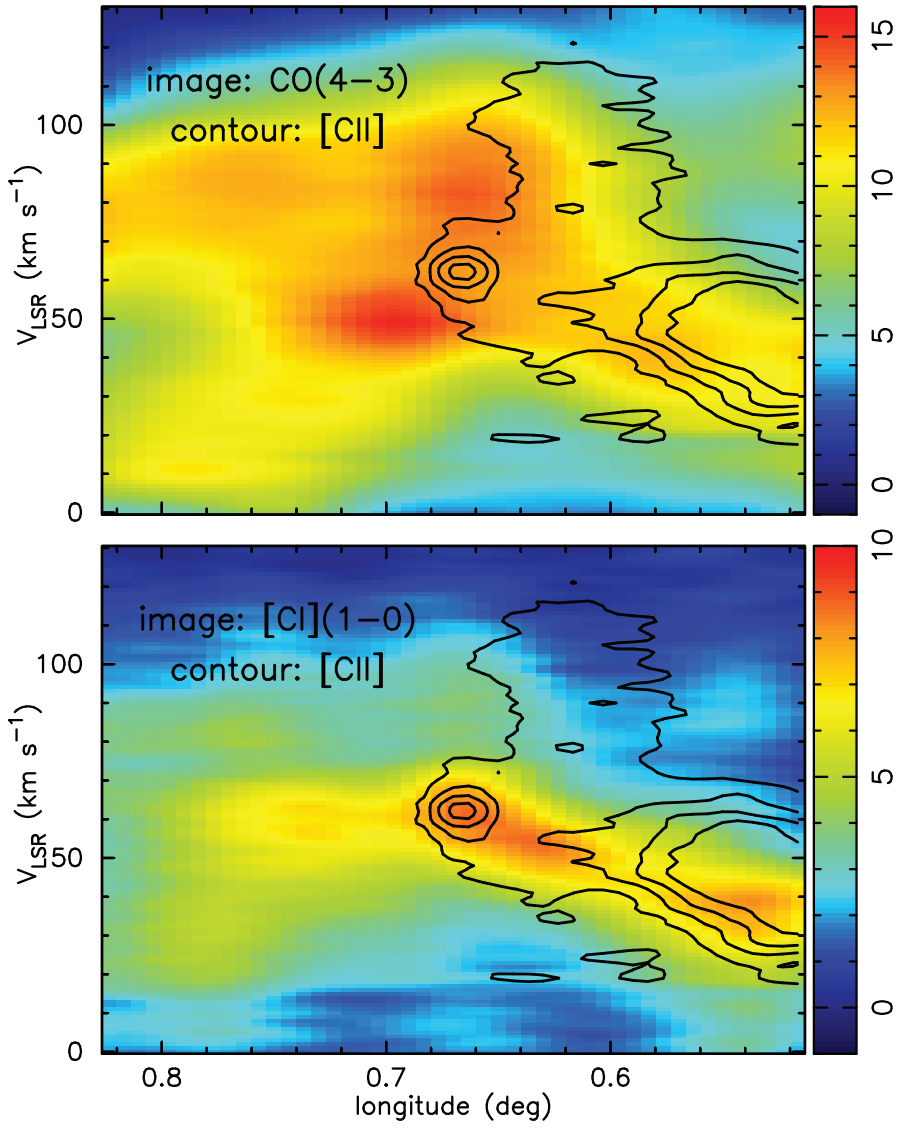

Fig. 11. Top: [C II $] l-V$ contour map including longitudes closest to Sgr B2 overlayed on an image of the AST/RO CO(4-3) emission. Bottom: same for [C II] overlayed on the [CI] image. The [C II] contours are in $T_{\mathrm{mb}}(\mathrm{K})$ and have the values $0.75,1.5,2.25$, and $3.0 \mathrm{~K}$. The values of $T_{\mathrm{mb}}(\mathrm{K})$ for the $\mathrm{CO}$ and $[\mathrm{CI}]$ images are given by the color bars on the right of each panel.

of its proximity to the open orbit gas streams (dashed line) in Fig. 4 we discuss this feature in more detail.

In Fig. 11 we show an expanded view of the $[\mathrm{C}$ II $]$ emission in this region compared to that of $[\mathrm{CI}]$ and $\mathrm{CO}(4-3)$ and in Fig. 12 we show their spectra at the peak, $(l, b)=(0.667,0 \circ 0)$. Near Sgr B2 the [C II] spectrum peaks at $V_{\mathrm{LSR}} \simeq 62 \mathrm{~km} \mathrm{~s}^{-1}$ with a linewidth $F W H M \simeq 14 \mathrm{~km} \mathrm{~s}^{-1}$, and is much narrower than $\mathrm{CO}(4-3)$, which has broad emission over $100 \mathrm{~km} \mathrm{~s}^{-1}$. The dip in the $\mathrm{CO}$ spectrum at this velocity could be due either to the superposition of many $\mathrm{CO}$ sources or absorption by lower excitation CO. In the models of Molinari et al. (2011) and Kruijssen et al. (2015) Sgr B2 is located at or near the tangent point of the streaming gas orbit so that many gas clouds would be expected to lie along this line-of-sight, but they would be spread over a range of distances. Thus the broad $\mathrm{CO}$ line is sampling many clouds along the line-of-sight in contrast to the [C II] line which appears to be strong only at the gas having a velocity associated with Sgr B2. The [CI] line also peaks at $V_{\mathrm{LSR}} \simeq 62 \mathrm{~km} \mathrm{~s}^{-1}$ but is broader then [C II], with a $F W H M \simeq 32 \mathrm{~km} \mathrm{~s}^{-1}$. Most likely the $[\mathrm{CI}$, as in the case of $\mathrm{CO}$, is coming from a number of gas clouds along the line of sight. In summary, while the bulk of the $\mathrm{CO}$ and fainter diffuse [C II] is associated with the open orbit gas streams, the compact [C II] is likely an ionized boundary layer or comes from H II regions associated with Sgr B2. 


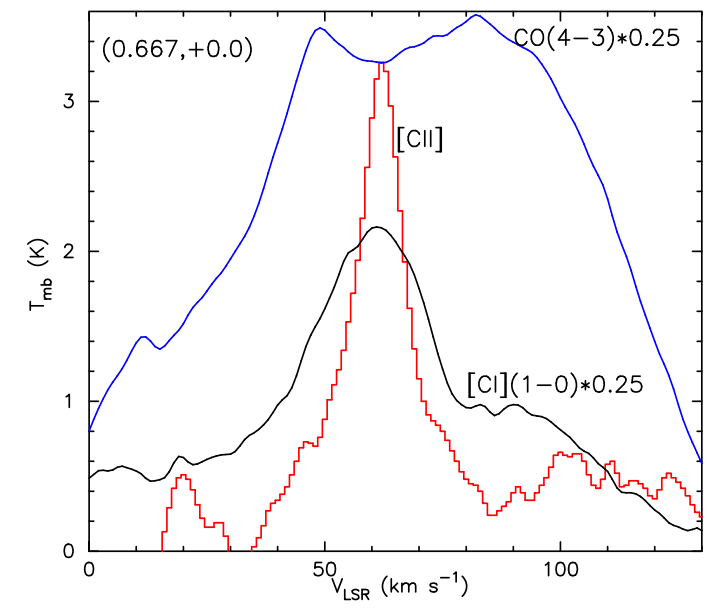

Fig. 12. [C II], $\mathrm{CO}(4-3)$, and $[\mathrm{CI}]$ spectra at the position of peak emission near Sgr B2, $(l, b)=(0.667,0 \circ 0)$, in the longitudinal scan.

\subsection{HII region $S 17$}

There is very strong compact [C II] emission at $b \sim 0.15$ in the latitude-velocity plots at $V_{\mathrm{LSR}} \sim-5 \mathrm{~km} \mathrm{~s}^{-1}$ as seen in Fig. $2 \mathrm{~d}$ and Fig. 4 (right panel). The peak temperature $T_{\mathrm{mb}}([\mathrm{C} \mathrm{II}]) \sim 35 \mathrm{~K}$ and the size of the emission region in $b$ is about 0.4 . The relative narrowness of the $[\mathrm{C} \mathrm{II}]$ spectra, $F W H M \sim 4$ to $5 \mathrm{~km} \mathrm{~s}^{-1}$, is more typical of clouds in the disk than the CMZ. The maps in Fig. 4 show that this region has weak $[\mathrm{CI}]$ and $\mathrm{CO}(4-3)$ emissions. We cannot be certain of the location of this H II source because with $V_{\mathrm{LSR}} \sim-5 \mathrm{~km} \mathrm{~s}^{-1}$ it could lie almost anywhere along the line of sight to the CMZ. However, the narrowness of the [C II] spectrum and the fact that it lines up almost exactly with an H II source in the WISE Catalog of Galactic H II regions (Anderson et al. 2014), suggests it is a local source associated with the large H II region designated S17 in the Sharpless cata$\log$ (Sharpless 1959), rather than arising from the CMZ.

\section{Summary}

We report on the first high spectral resolution OTF strip scans of [C II] $(158 \mu \mathrm{m})$ across the CMZ taken with Herschel HIFI as part of the GOT C+ open time key programme. A primary goal of this survey was to explore what types of features within the $\mathrm{CMZ}$ can be detected in $[\mathrm{C} \mathrm{II}]$ and what can be learned about the kinematics and properties of the gas containing $\mathrm{C}^{+}$. This survey consisted of two OTF strip scans, each 1.6 long centered on $\left(0^{\circ}, 0^{\circ}\right)$, one in longitude and the other in latitude. The effective spatial resolution of this survey was $\sim 80^{\prime \prime}$ along the scan and $12^{\prime \prime}$ perpendicular to the scan. [C II] was detected in a wide variety of CMZ features including G0.253+0.016 (the Brick), the open orbit streams of gas, the Radio Arc, the arched filaments, and the gas near Sgr A and Sgr B2.

We found that $[\mathrm{CII}]$ traces portions of the several open orbit streams of cloud-forming gas (cf. Longmore et al. 2013; Kruijssen et al. 2015) that stretch over 200 pc across the CMZ (Tsuboi et al. 1999; Molinari et al. 2011). A fit to the spatialvelocity longitudinal cut of $[\mathrm{CII}]$ through $b=0^{\circ}$ yields orbital velocities up to $100 \mathrm{~km} \mathrm{~s}^{-1}$ and a major axis $\sim 120 \mathrm{pc}$, consistent with the open orbit stream models of Kruijssen et al. (2015). We also find that [C II], [CI], and $\mathrm{CO}(4-3)$ may trace the corrugated velocity features of dense gas in the streams studied by Henshaw et al. (2016a), but we are unable to draw any conclusions about the relative motion of these outer cloud gas tracers with respect to those of the dense cores.

[C II] emission is detected across the Brick. There are two strong emission velocity features at $l \sim 0.21667$ located outside the dense core and CO image of the Brick's molecular gas, one of which is associated with the Brick. The other feature is associated with another cloud at higher velocities that has $[\mathrm{CI}]$ and $\mathrm{CO}$ but no evidence of the dense gas tracer $\mathrm{HN}^{13} \mathrm{C}$. Both [C II] features likely originate in an ionized boundary layer or extended $\mathrm{H}$ II region. We estimated the electron density in these two regions to be about 100 to $140 \mathrm{~cm}^{-3}$ assuming the [C II] arises from a highly ionized gas. We also analyze the conditions where the [C II] longitudinal strip scan intersects the edge of the Brick by fitting the line parameters and calculating column densities of [C II], [C I], $\mathrm{CO}(4-3)$, and $\mathrm{HN}^{13} \mathrm{C}$ at $\mathrm{G} 0.250+0.0$. We find that the $\mathrm{HNC}$ column density is about $10 \%$ of the value through the core, and that $\mathrm{C}^{+}$forms an envelope around the molecular core about $0.8 \mathrm{pc}$ in depth.

We detect two prominent [C II] features at $l \sim 0.06$ at velocities, $V_{\mathrm{LSR}} \sim-34$ to $-43 \mathrm{~km} \mathrm{~s}^{-1}$ corresponding to the Radio Arc near the base of the arched filament. Assuming that the [C II] from this region arises primarily from fully ionized gas we estimate that the electron densities, $n(\mathrm{e}) \sim 100$ to $130 \mathrm{~cm}^{-3}$ at the base of the thermal arched filaments.

The OTF strip scans intersect the warm dust bubble as seen in projection as a doughnut, identified in the Herschel HI-GAL infrared survey (Molinari et al. 2014). [C II] emission appears to trace this warm dust, which is not surprising as the UV which keeps the dust and gas warm also gives rise to $\mathrm{C}^{+}$and, therefore, [C II]. We do not have sufficient information to know for certain whether the source of [C II] is from PDRs, CO-dark $\mathrm{H}_{2}$, or fully ionized gas. However, an analysis of the intensity ratio of [C II] to $\mathrm{CO}(4-3)$ and $[\mathrm{CI}](1-0)$ indicates that a significant fraction of the $\left[\mathrm{C}\right.$ II] in the inner $l= \pm 0.25$ along $b=0^{\circ}$ arises from ionized gas. The observed velocity structure in the [C II] emission is consistent with the kinematics of an expanding bubble. Finally, we detect only weak [C II] emission in the strip scan that passes within 4 to $5 \mathrm{pc}$ (in projection) from $\mathrm{Sgr} \mathrm{B}$, an active region of star formation with strong compact H II sources.

In summary the GOT C+ [C II] survey of the CMZ, although limited to two narrow OTF strip scans, shows that the bright farIR $158 \mu \mathrm{m} \mathrm{C}^{+}$line, when observed with high spatial and spectral resolution, brings out a wide range of emission features tracing a variety of physical conditions in the interstellar gas in the $\mathrm{CMZ}$ and near the Galactic center. The GOT C+ strip scans demonstrate the need for future large-scale, spectrally resolved [C II] $l-b$ maps, fully sampled in in both longitude and latitude. Such maps, as well as those of other ions, such as [N II], will be a vital and important template for understanding galactic nuclei.

Acknowledgements. We would like to thank Dr. David Teyssier for clarifications regarding the use of the hebCorrection tool. We also thank an anonymous referee for numerous in depth comments and suggestions that improved the analysis and discussion significantly. This work was performed at the Jet Propulsion Laboratory, California Institute of Technology, under contract with the National Aeronautics and Space Administration. US Government sponsorship acknowledged.

\section{References}

Anderson, L. D., Bania, T. M., Balser, D. S., et al. 2014, ApJS, 212, 1 Ao, Y., Henkel, C., Menten, K. M., et al. 2013, A\&A, 550, A135 Bennett, C. L., Fixsen, D. J., Hinshaw, G., et al. 1994, ApJ, 434, 587 Bergin, E. A., Goldsmith, P. F., Snell, R. L., \& Langer, W. D. 1997, ApJ, 482, 285 
Bertram, E., Glover, S. C. O., Clark, P. C., Ragan, S. E., \& Klessen, R. S. 2016, MNRAS, 455, 3763

Clark, P. C., Glover, S. C. O., Ragan, S. E., Shetty, R., \& Klessen, R. S. 2013, ApJ, 768, L34

Dame, T. M., Hartmann, D., \& Thaddeus, P. 2001, ApJ, 547, 792

de Graauw, T., Helmich, F. P., Phillips, T. G., et al. 2010, A\&A, 518, L6

Enokiya, R., Torii, K., Schultheis, M., et al. 2014, ApJ, 780, 72

Ferrière, K., Gillard, W., \& Jean, P. 2007, A\&A, 467, 611

García, P. 2015, Ph.D. Thesis, University of Cologne, Germany, http://kups.ub.uni-koeln.de/6358/

García, P., Simon, R., Stutzki, J., et al. 2016, A\&A, 588, A131

Ginsburg, A., Henkel, C., Ao, Y., et al. 2016, A\&A, 586, A50

Goldsmith, P. F., Langer, W. D., Pineda, J. L., \& Velusamy, T. 2012, ApJS, 203, 13

Goldsmith, P. F., Yıldız, U. A., Langer, W. D., \& Pineda, J. L. 2015, ApJ, 814 133

Güsten, R. 2007, KPGT_rguesten_1: The HEXGAL (Herschel EXtraGALactic) Key Project: Physical and Chemical Conditions of the ISM in Galactic Nuclei, Herschel Space Observatory Proposal, 45

Henshaw, J. D., Longmore, S. N., \& Kruijssen, J. M. D. 2016a, MNRAS, 463 L122

Henshaw, J. D., Longmore, S. N., Kruijssen, J. M. D., et al. 2016b, MNRAS, 457,2675

Jackson, J. M., Heyer, M. H., Paglione, T. A. D., \& Bolatto, A. D. 1996, ApJ, 456, L91

Jones, P. A., Burton, M. G., Cunningham, M. R., et al. 2012, MNRAS, 419, 2961

Koyama, K., Hyodo, Y., Inui, T., et al. 2007, PASJ, 59, 245

Kruijssen, J. M. D., Dale, J. E., \& Longmore, S. N. 2015, MNRAS, 447, 1059

Lang, C. C., Goss, W. M., \& Morris, M. 2001, AJ, 121, 2681

Lang, C. C., Goss, W. M., \& Morris, M. 2002, AJ, 124, 2677

Langer, W. D., \& Pineda, J. L. 2015, A\&A, 580, A5

Langer, W. D., Velusamy, T., Pineda, J. L., et al. 2010, A\&A, 521, L17

Langer, W. D., Velusamy, T., Pineda, J. L., Willacy, K., \& Goldsmith, P. F. 2014, A\&A, 561, A122

Langer, W. D., Goldsmith, P. F., Pineda, J. L., et al. 2015, A\&A, 576, A1

Langer, W. D., Goldsmith, P. F., \& Pineda, J. L. 2016, A\&A, 590, A43

Lis, D. C., Menten, K. M., Serabyn, E., \& Zylka, R. 1994, ApJ, 423, L39

Longmore, S. N., Rathborne, J., Bastian, N., et al. 2012, ApJ, 746, 117

Longmore, S. N., Kruijssen, J. M. D., Bally, J., et al. 2013, MNRAS, 433, L15

Longmore, S. N., Kruijssen, J. M. D., Bastian, N., et al. 2014, Protostars and Planets VI, 291

Mangum, J. G., Emerson, D. T., \& Greisen, E. W. 2007, A\&A, 474, 679

Martin, C. L., Walsh, W. M., Xiao, K., et al. 2004, ApJS, 150, 239

Mills, E. A. C., \& Morris, M. R. 2013, ApJ, 772, 105

Mills, E. A. C., Butterfield, N., Ludovici, D. A., et al. 2015, ApJ, 805, 72

Molinari, S., Bally, J., Noriega-Crespo, A., et al. 2011, ApJ, 735, L33
Molinari, S., Bally, J., Glover, S., et al. 2014, Protostars and Planets VI, 125 Morris, M. 1997, in IAU Symp. 170, eds. W. B. Latter, S. J. E. Radford, P. R. Jewell, J. G. Mangum, \& J. Bally, 57

Morris, M., \& Serabyn, E. 1996, ARA\&A, 34, 645

Muno, M. P., Bauer, F. E., Baganoff, F. K., et al. 2009, ApJS, 181, 110

Nakagawa, T., Yui, Y. Y., Doi, Y., et al. 1998, ApJS, 115, 259

Oberst, T. E., Parshley, S. C., Nikola, T., et al. 2011, ApJ, 739, 100

Oka, T., Hasegawa, T., Sato, F., Tsuboi, M., \& Miyazaki, A. 1998, ApJS, 118 455

Oka, T., Nagai, M., Kamegai, K., \& Tanaka, K. 2011, ApJ, 732, 120

Oka, T., Onodera, Y., Nagai, M., et al. 2012, ApJS, 201, 14

Pilbratt, G. L., Riedinger, J. R., Passvogel, T., et al. 2010, A\&A, 518, L1

Pillai, T., Kauffmann, J., Tan, J. C., et al. 2015, ApJ, 799, 74

Pineda, J. L., Velusamy, T., Langer, W. D., et al. 2010, A\&A, 521, L19

Pineda, J. L., Langer, W. D., Velusamy, T., \& Goldsmith, P. F. 2013, A\&A, 554 A103

Pineda, J. L., Langer, W. D., \& Goldsmith, P. F. 2014, A\&A, 570, A121

Ponti, G., Morris, M. R., Terrier, R., \& Goldwurm, A. 2013, in Cosmic Rays in Star-Forming Environments, eds. D. F. Torres, \& O. Reimer, Astrophys. Space Sci. Proc., 34, 331

Ponti, G., Morris, M. R., Terrier, R., et al. 2015, MNRAS, 453, 172

Porquet, D., Rodriguez, J., Corbel, S., et al. 2003, A\&A, 406, 299

Rathborne, J. M., Longmore, S. N., Jackson, J. M., et al. 2014, ApJ, 786, 140

Riegel, K. W., \& Crutcher, R. M. 1972, A\&A, 18, 55

Schöier, F. L., van der Tak, F. F. S., van Dishoeck, E. F., \& Black, J. H. 2005 , A\&A, 432, 369

Serabyn, E., \& Guesten, R. 1987, A\&A, 184, 133

Serabyn, E., \& Morris, M. 1994, ApJ, 424, L91

Sharpless, S. 1959, ApJS, 4, 257

Simpson, J. P., Colgan, S. W. J., Cotera, A. S., et al. 2007, ApJ, 670, 1115

Taylor, G. B., Morris, M., \& Schulman, E. 1993, AJ, 106, 1978

Tsuboi, M., Handa, T., \& Ukita, N. 1999, ApJS, 120, 1

van der Tak, F. F. S., Black, J. H., Schöier, F. L., Jansen, D. J., \& van Dishoeck, E. F. 2007 , A\&A, 468, 627

Velusamy, T., \& Langer, W. D. 2014, A\&A, 572, A45

Velusamy, T., Langer, W. D., Pineda, J. L., et al. 2010, A\&A, 521, L18

Velusamy, T., Langer, W. D., Pineda, J. L., \& Goldsmith, P. F. 2012, A\&A, 541, L10

Velusamy, T., Langer, W. D., Goldsmith, P. F., \& Pineda, J. L. 2015, A\&A, 578, A135

Wang, Q. D., Gotthelf, E. V., \& Lang, C. C. 2002, Nature, 415, 148

Wolfire, M. G., Hollenbach, D., \& McKee, C. F. 2010, ApJ, 716, 1191

Yusef-Zadeh, F., \& Morris, M. 1987, ApJ, 322, 721

Yusef-Zadeh, F., Morris, M., \& Chance, D. 1984, Nature, 310, 557

Yusef-Zadeh, F., Roberts, D. A., Goss, W. M., Frail, D. A., \& Green, A. J. 1999, ApJ, 512, 230 